\title{
Multirotor wind turbine wakes
}

Cite as: Phys. Fluids 31, 085106 (2019); https://doi.org/10.1063/1.5097285

Submitted: 24 March 2019 . Accepted: 11 July 2019 . Published Online: 06 August 2019

Majid Bastankhah (D), and Mahdi Abkar (iD)
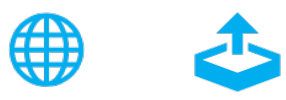

\section{ARTICLES YOU MAY BE INTERESTED IN}

Wind farm power optimization via yaw angle control: A wind tunnel study

Journal of Renewable and Sustainable Energy 11, 023301 (2019); https://

doi.org/10.1063/1.5077038

Influence of atmospheric stability on wind-turbine wakes: A large-eddy simulation study

Physics of Fluids 27, 035104 (2015); https://doi.org/10.1063/1.4913695

Conditional dynamic subfilter modeling

Physics of Fluids 31, 085107 (2019); https://doi.org/10.1063/1.5098813

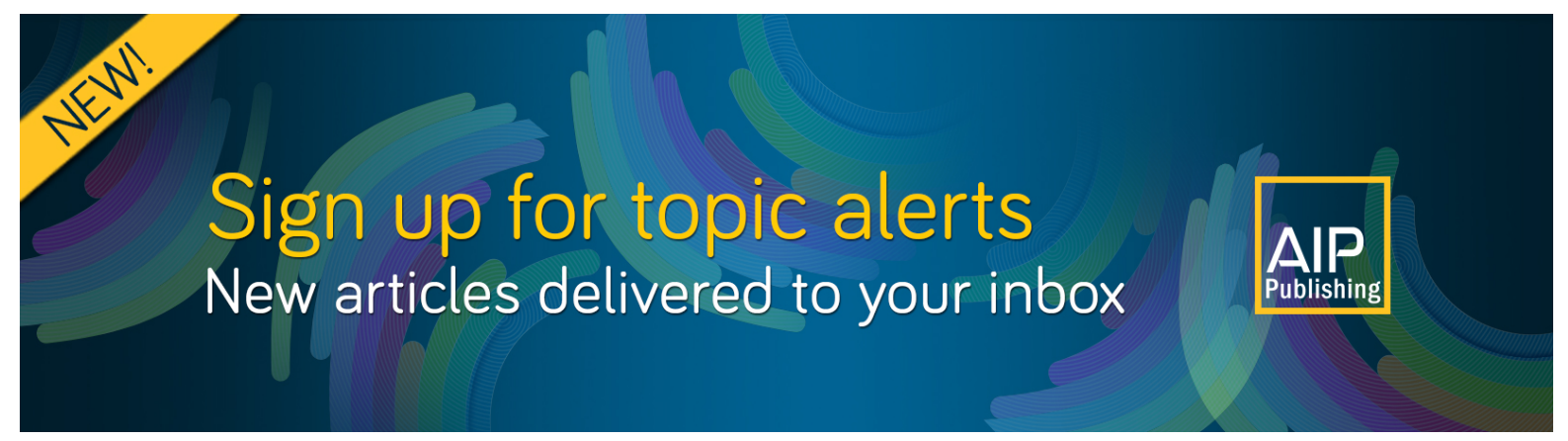




\title{
Multirotor wind turbine wakes
}

\author{
Cite as: Phys. Fluids 31, 085106 (2019); doi: 10.1063/1.5097285 \\ Submitted: 24 March 2019 • Accepted: 11 July 2019 • \\ Published Online: 6 August 2019
}

Majid Bastankhah $^{1, a)}$ (D) and Mahdi Abkar ${ }^{2, b)}$ (D)

\author{
AFFILIATIONS \\ ${ }^{1}$ Department of Engineering, Durham University, Durham DH1 3LE, United Kingdom \\ ${ }^{2}$ Department of Engineering, Aarhus University, 8000 Aarhus C, Denmark
}

a) Electronic mail: majid.bastankhah@durham.ac.uk

b) Electronic mail: abkar@eng.au.dk

\begin{abstract}
To fulfill the increasing need for large power generation by wind turbines, the concept of multirotor wind turbines has recently received attention as a promising alternative to conventional massive single-rotor wind turbines. To shed light on the viability of this concept, largeeddy simulation is employed in this study to compare wake flow properties of a multirotor wind turbine with those of a single-rotor turbine. The wake of a multirotor turbine is found to recover faster at short downwind distances, where the whole wake is characterized as an array of localized high velocity-deficit regions associated with each rotor. However, as the wake moves downstream, rotor wakes start interacting with each other until they eventually form a single wake. This transition from a wake array to a single wake adversely affects the initial fast recovery of multirotor turbine wakes. A budget analysis of mean kinetic energy is performed to analyze the energy transport into the wake before and after this transition. In addition, the effect of different geometrical configurations on wake characteristics of a multirotor turbine was examined. We found that the recovery rate of multirotor turbine wakes is enhanced by the increase in rotor spacing, whereas the number and rotation direction of rotors do not play a significant role in the wake recovery. A simple analytical relationship is also developed to predict the streamwise distance at which the transition from a wake array to a single wake occurs for multirotor wind turbines.
\end{abstract}

Published under license by AIP Publishing. https://doi.org/10.1063/1.5097285

\section{INTRODUCTION}

In order to increase the power generated by each wind turbine, the size of utility-scale wind turbines has been steadily increasing over the last few decades. Although the use of these massive turbines results in higher energy production, they are associated with high manufacturing costs. Moreover, shipment, as well as the maintenance of such large rotors, have proved to be formidable challenges, in particular, for offshore installations. To address this problem, there is a new interest in the use of multiple small rotors that are installed on a single support structure, instead of a single big rotor used in conventional turbines. ${ }^{1}$ Several structural and economical benefits of such turbines have been identified in the literature. ${ }^{2-}$ Prior studies ${ }^{5-7}$ have estimated a cost reduction of more than $15 \%$ for a multimegawatt multirotor turbine compared to a single-rotor turbine with the same rated power. Despite the potential advantage of multirotor turbines, little is known about the complex interaction of multirotor wakes, which occurs immediately behind these turbines. The understanding of multirotor turbine wakes is both practically and fundamentally of importance. Apart from practical implications of multirotor turbines in future wind farm projects, studies of their wake flows can provide valuable physical insights into the complex interaction of wake flows generated by several side-by-side objects. ${ }^{8,9}$ Note that multirotor turbines discussed in this paper are conceptually different from coaxial rotors, commonly known as dual rotors, which have received a great deal of attention in the wind energy research community over the past few years (see, for instance, Refs. 10 and 11, among others). Unlike dual rotors, multirotor turbines discussed here consist of multiple rotors that are installed next to each other on the same plane.

Among the few studies addressing wakes of multirotor wind turbines, Ghaisas et al. ${ }^{12}$ used large-eddy simulations (LESs) coupled with the standard actuator-disk model to compare the wake of a multirotor turbine with the one of a conventional turbine comprised of a single rotor per tower. They showed that the multirotor turbine wake recovers faster, while the turbulence intensity in the wake is smaller, compared to the wake of a single-rotor turbine. They also studied wake flows in a row of five multirotor wind turbines, aligned with the incoming wind, and found a higher power efficiency for the multirotor wind farm compared to a single-rotor 
wind farm. However, in their simulations, a relatively large value of spacing between the rotors (equal to one rotor diameter) was considered. Moreover, due to the representation of turbines using the standard actuator-disk model, effects of turbine-induced flow rotation were dismissed. Recently, van der Laan et al. ${ }^{13}$ have studied the wake flow behind the multirotor turbine demonstrator installed by Vestas Wind Systems A/S. In particular, using Reynolds-averaged Navier-Stokes (RANS) simulations, they showed that the multirotor wind turbine wake recovers faster compared to the wake of a single-rotor wind turbine with an equivalent rotor diameter.

Despite the promising findings reported in the abovementioned studies, important remaining challenges are to understand the physics of wake formation and recovery behind multirotor wind turbines. In addition, the effects of rotor numbers, size, spacing, and rotation on turbine wake characteristics remain to be elucidated. In this study, LES is employed to study in detail the wake of a multirotor wind turbine. The main focus of the present work is to give a detailed comparison of the wake of a four-rotor wind turbine with the one of a single-rotor turbine. Moreover, a parametric study is carried out to examine wakes of multirotor turbines with different geometrical configurations (e.g., rotor spacing, rotor number, and rotation direction). The rest of the paper is organized as follows. In Sec. II, a summary of the LES framework is provided. Results are presented in Sec. III, and Sec. IV summarizes main findings of this paper.

\section{LARGE-EDDY SIMULATION FRAMEWORK}

The validated LES code used here solves the filtered NavierStokes equations for incompressible turbulent flow as

$$
\begin{gathered}
\frac{\partial \tilde{u}_{i}}{\partial x_{i}}=0 \\
\frac{\partial \tilde{u}_{i}}{\partial t}+\tilde{u}_{j} \frac{\partial \tilde{u}_{i}}{\partial x_{j}}=-\frac{1}{\rho_{o}} \frac{\partial \tilde{p}}{\partial x_{i}}-\frac{\partial \tau_{i j}}{\partial x_{j}}-\frac{f_{i}}{\rho_{o}}
\end{gathered}
$$

where $\left(\tilde{u}_{1}, \tilde{u}_{2}, \tilde{u}_{3}\right)=(\tilde{u}, \tilde{v}, \tilde{w})$ are the components of the filtered velocity field and $i=1,2$, and 3 , respectively, refer to the streamwise $x$, spanwise $y$, and wall-normal $z$ directions. Time is denoted by $t, \tilde{p}$ is the filtered pressure, $\rho_{o}$ is the fluid density, $\tau_{i j}$ is the subgrid-scale stress tensor, and $f_{i}$ is a body force representing the effect of wind turbines on the flow field. The code uses pseudospectral discretization in the streamwise and spanwise directions and a second-order finite difference scheme in the wall-normal direction. A secondorder Adam-Bashforth method is used for the time advancement. The viscous forces are neglected in the governing equation since the Reynolds number of the atmospheric boundary-layer flow is very high. The Lagrangian scale-dependent Smagorinsky model is also utilized to parameterize the subgrid-scale turbulent motions. ${ }^{14,15}$ The presented LES framework has been well validated and used in earlier wind-energy research publications. The reader may refer to Refs. 16-19 for a more detailed description of the LES framework and the solver.

The inflow condition is generated using a precursor technique and by simulating a neutrally stratified boundary layer over flat terrain. The computational domain is $1600 \mathrm{~m} \times 800 \mathrm{~m} \times 355 \mathrm{~m}$ in the $x$, $y$, and $z$ directions, respectively, and it is discretized uniformly into $160 \times 160 \times 72$ computational nodes. The boundary layer is driven by an imposed pressure gradient. The aerodynamic surface roughness is $0.005 \mathrm{~m}$. The wall shear stress is computed according to the standard log law. ${ }^{14,20}$ In wake flow simulations, a fringe zone is used to adjust the flow from the downstream wake state to an undisturbed inflow condition. ${ }^{21-23}$ The vertical profiles of mean wind speed and turbulence intensity in the streamwise direction obtained from the precursor simulation are shown in Fig. 1. The inflow has a mean hub-height velocity $\bar{u}_{h}$ of about $8 \mathrm{~m} / \mathrm{s}$, where the overbar denotes time averaging. The turbulence intensity $I=\sigma_{u} / \bar{u}_{h}$ at the hub height is about $6.7 \%$, where $\sigma_{u}$ is the standard deviation of the streamwise velocity.

The turbine-induced forces are parametrized using the rotating actuator-disk model. ${ }^{16}$ Unlike the standard actuator-disk model, the rotating actuator-disk technique takes into account the effects of turbine-induced flow rotation and nonuniform distribution of forces. $^{24}$ The aerodynamic forces acting on the blades are determined using the relative wind velocity, the geometry of the blade airfoil, and the lift and drag characteristics of the blade obtained from the tabulated airfoil data. More details on the rotating actuatordisk model and its implementation and validation in the LES code can be found in Refs. 16 and 24-26.

A schematic depicted in Fig. 2 shows the nomenclature used in this paper to describe geometrical information of single-rotor [henceforth referred to as single-rotor (SR)] and multirotor (henceforth referred to as MR) wind turbines. The diameter and hub height of the SR turbine are denoted by $D$ and $z_{h}$, respectively. For MR turbines, each rotor with a diameter denoted by $d$ is spaced by $s$ from adjacent rotors. For the sake of simplicity, rotors are assumed to be spaced equally in the horizontal and vertical directions in this paper. In order to have equal frontal areas for a SR and a MR turbine,

$$
d=\frac{D}{\sqrt{n}}
$$

where $n$ is the number of rotors. While the MR turbine, shown in Fig. 2, has four rotors, MR turbines with different numbers of rotors
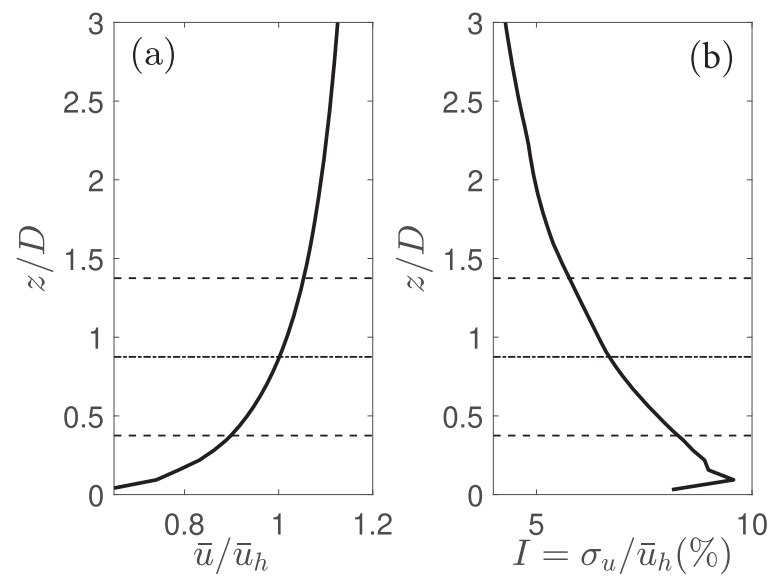

FIG. 1. Vertical profiles of (a) the time-averaged streamwise velocity $\bar{u}$, normalized by $\bar{u}_{h}$, and (b) the turbulence intensity $I=\sigma_{u} / \bar{u}_{h}(\%)$ for the incoming flow. Horizontal lines indicate the turbine hub height as well as the top and bottom rotor tips. 
(a)

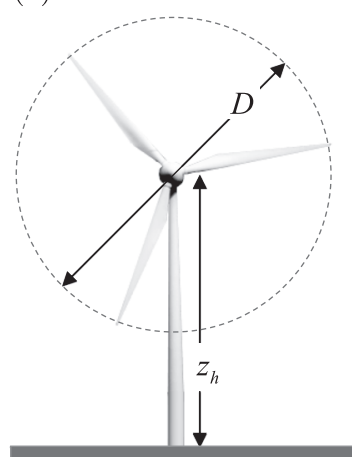

(b)

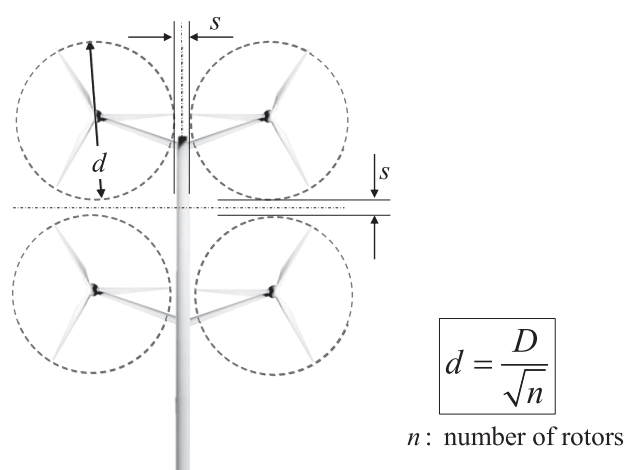

FIG. 2. Schematic figure of (a) a single-rotor (SR) wind turbine and (b) a multirotor (MR) wind turbine. Note that the turbine towers shown are for illustrative purposes only, and they are not modeled in the simulations. $n$ will be discussed in Sec. III D. To differentiate between these different cases, we, later on, use the term $\mathrm{MR} n$, which refers to a MR case with $n$ rotors. Moreover, to support some of the provided arguments, we occasionally discuss wakes of SRs with a hub height of $z_{h}$ but a rotor diameter equal to the one of a MRn turbine [recall from Eq. (3): $d=D / \sqrt{n}$ for a MR $n$ turbine]. To distinguish these cases from the base SR case with $D$, the term $\mathrm{SR} n$ is hereafter used to denote a SR case with a rotor diameter equal to $D / \sqrt{n}$.

The SR wind turbine simulated in this study is the Vestas V80$2 \mathrm{MW}$ wind turbine with $D=80 \mathrm{~m}$ and $z_{h}=70 \mathrm{~m}$. Details of the Vestas V80-2MW wind turbine in a simulation of an operational wind farm can be found in Refs. 25 and 26. For smaller SR turbines (i.e., SRn turbines with $n>1$ ) and rotors of MR turbines, scaled-down versions of the Vestas V80 rotor with the same blade properties (e.g., identical normalized chord and twist distributions) are adopted. All of the simulated cases are found to have relatively similar values of the thrust coefficient $C_{T}$, falling within the range of $0.8 \pm 0.02$.

Overall, in order to study different aspects of MR turbine aerodynamics, 14 different turbines as well as their wake flows are numerically simulated in this paper. A detailed wake comparison of a MR4 wind turbine with a SR one is provided in Sec. III A. Wake flow distributions for MR4 turbines with three different values of rotor spacing are examined in Sec. III B. The effect of rotor size on wake characteristics is studied in Sec. III C by simulating four SR turbines with different rotor diameters. In Sec. III D, four MR turbines with different numbers of rotors are simulated to study how MR turbine wakes are affected by numbers of rotors. Finally, the impact of rotor rotation on MR turbine wake structures is investigated for six MR turbines with different distributions of the rotation direction in Sec. III E.

\section{RESULTS}

\section{A. Detailed comparison of turbine wakes: MR vs SR turbines}

In the following, a detailed comparison of the wake of a SR turbine with the one of a MR4 turbine, schematically shown in Fig. 2, is presented. Note that the turbine towers shown in Fig. 2 are for illustrative purposes only, and they are not modeled in the simulations. Since the value of $D$ is selected to be $80 \mathrm{~m}$ in this paper, based on Eq. (3), $d$ should be equal to $40 \mathrm{~m}$ for the MR4 case. The value of rotor spacing $s$ is selected to be $0.1 d$, inspired by the multirotor turbine concept demonstrator previously installed by Vestas Wind Systems A/S at DTU Risø campus.

Although the main motive of this paper is to explore the farwake region of MR turbines, first, we briefly discuss the flow distribution immediately behind the turbine. The flow distribution in the near wake serves as an initial condition for the far-wake region. Therefore, prior to focusing on the far-wake region, it is useful to investigate the minimum value of the streamwise velocity in the near wake. Figure 3(a) shows the variation of $\langle\bar{u}\rangle$, normalized by $\bar{u}_{h}$, as wind passes through the wind turbine for both the MR4 and SR cases, where $\langle\cdot\rangle$ denotes spatial averaging over the rotor disk frontal area. Downstream of the turbine, according to Bernoulli's principle, the recovery of the air pressure leads to a further reduction in the streamwise velocity until the wind speed eventually reaches a minimum value, where the air pressure in the wake equals the one in the surroundings. It is noteworthy to mention that the minimum streamwise velocity value in the near wake of the MR4 turbine is different from the one for the SR turbine. This difference is shown in Fig. 3(b), which plots the variation of the spatially averaged velocity deficit $\langle\Delta \bar{u}\rangle$, normalized by its value at the rotor plane $\left\langle\Delta \bar{u}_{d}\right\rangle$. The velocity deficit $\Delta \bar{u}(x, y, z)$ is defined as $\bar{u}_{i n}(y, z)-\bar{u}(x$, $y, z)$, where $\bar{u}_{\text {in }}$ is the time-averaged incoming velocity. As seen in Fig. 3(b), the maximum velocity deficit in the wake of a MR4 turbine is lower than the one of the SR turbine. In order to better investigate this discrepancy, the results for a single rotor with a diameter of $D / \sqrt{4}$ (i.e., SR4) is also reported in the figure. As shown, the maximum velocity deficit for the MR4 case is smaller than the singlerotor case regardless of its rotor diameter size. Interestingly, this discrepancy can also be seen in the results reported in the previous works. ${ }^{12,13}$ We hypothesize that a low value of the maximum velocity deficit for rotors in the MR4 case is due to local blockage effects exerted by the adjacent rotors. This is elaborated in the following.

For a rotor in an infinite medium, the well-known Betz theory for an ideal streamtube passing through the rotor suggests that $\Delta \bar{u}_{\max } / \Delta \bar{u}_{d}$ is equal to 2 . When a rotor is placed in a finite medium, however, the blockage caused by the rotor accelerates the surrounding flow (i.e., bypass flow), which in turn can affect the wake structure. According to the model originally developed by Garrett and Cummins $^{27}$ for tidal turbines, one can write the below relationship 
(a)
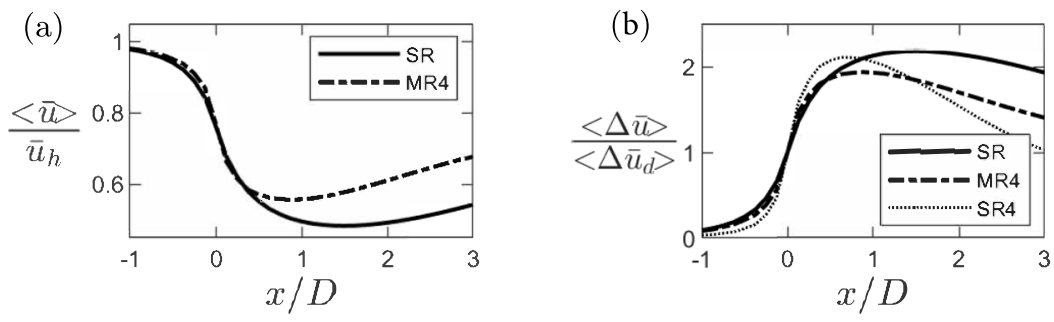

(c)

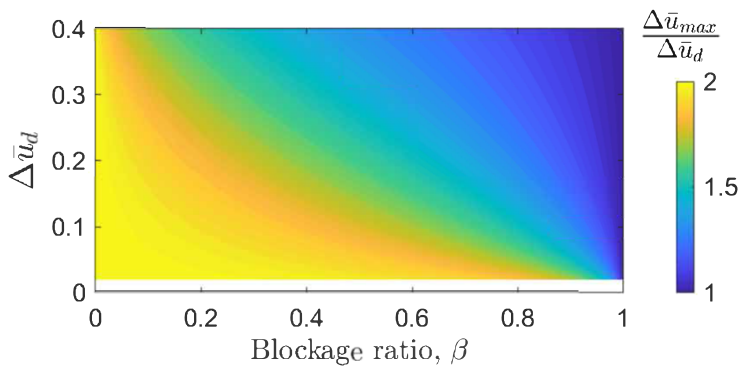

FIG. 3. (a) Variation of the spatially averaged streamwise velocity $\langle\bar{u}\rangle$, normalized by $\bar{u}_{h}$, as wind passes through the wind turbine, (b) variation of the spatially averaged velocity deficit $\langle\Delta \bar{u}\rangle$, normalized by its value at the rotor plane $\left\langle\Delta \bar{u}_{d}\right\rangle$, and (c) variation of $\Delta \bar{u}_{\max } / \Delta \bar{u}_{d}$ predicted by Eq. (4) as a function of $\beta$ and $\Delta \bar{u}_{d}$. for a streamtube passing through a rotor in a finite medium ${ }^{28}$

$$
\begin{aligned}
& \beta\left(\Delta \bar{u}_{d}-1\right)\left[\Delta \bar{u}_{d}-2\left(\Delta \bar{u}_{\text {max }}-1\right)^{2}+\left(3 \Delta \bar{u}_{\text {max }}-3\right)\left(\Delta \bar{u}_{d}-1\right)-1\right] \\
& -\left(\Delta \bar{u}_{\text {max }}-1\right)^{2}\left(2 \Delta \bar{u}_{d}-\Delta \bar{u}_{\text {max }}\right)=0,
\end{aligned}
$$

where $\beta$ is the blockage ratio defined as the rotor frontal area to the medium cross section. Note that the above equation is reduced to $\Delta \bar{u}_{\max }=2 \Delta \bar{u}_{d}$ for $\beta$ equal to zero. Figure 3(c) shows the variation of $\Delta \bar{u}_{\text {max }} / \Delta \bar{u}_{d}$ predicted by Eq. (4) as a function of $\beta$ and $\Delta \bar{u}_{d}$. The figure shows that the value of $\Delta \bar{u}_{\text {max }} / \Delta \bar{u}_{d}$ is significantly reduced by an increase in $\beta$, especially for cases with high values of $\Delta \bar{u}_{d}$ (i.e., high values of $C_{T}$ ). For the extreme case when $\beta$ is equal to one (i.e., the turbine occupies the whole flow cross section), $\Delta \bar{u}_{\text {max }}$ is the same as $\Delta \bar{u}$, which is expected according to the conservation of mass. As pointed out by the works of Nishino and his co-authors (e.g., Refs. 29 and 30), the model expressed by Eq. (4) is a one-dimensional model that is unable to distinguish between the so-called local and global blockage effects. The global blockage is referred to the blockage of the turbine with respect to the whole flow cross section, while the local blockage concerns the local effect of each rotor on neighboring ones. For the numerical setup, the global blockage effects are very small and similar for both turbines ( $\beta$ is equal to 0.0177 for both SR and MR4 cases). However, rotors experience local blockage effects in the MR4 case as they are placed in close proximity of each other. Since each rotor is confined by the neighboring rotors only from three sides, the local value of $\beta$ in Eq. (4) cannot be determined, and therefore it is impossible to quantify local blockage effects using this equation. However, Fig. 3(c) confirms that the flow blockage essentially reduces the value of $\Delta \bar{u}_{\max } / \Delta \bar{u}_{d}$, which is in agreement with our LES data shown in Fig. 3(b).

We now turn our attention to the wake flow distribution further downstream. Figure 4 shows contours of the normalized velocity deficit $\Delta \bar{u} / \bar{u}_{h}$ in vertical planes, normal to the incoming flow, at different downwind locations for both the SR and the MR4 turbines. To facilitate quantitative comparison between the wakes of the two turbines, Fig. 5 shows lateral and vertical profiles of the normalized velocity deficit $\Delta \bar{u} / \bar{u}_{h}$ at several downwind locations. Note that, for the MR4 case, the center of rotors has vertical and horizontal offsets with a magnitude of $(d+s) / 2$ with respect to the center of the SR. In order to better compare the wakes, therefore, velocity deficit profiles are plotted along two vertical lines [i.e., $y=0$, and $y=-(d$ $+s) / 2$ ] and two horizontal lines [i.e., $z=z_{h}$ and $z=z_{h}-(d+s) / 2$ ]. At short downwind distances (e.g., $x=2 D$ ), as seen in both Figs. 4 and 5 , the flow downwind of the MR turbine is characterized by four localized, high velocity deficit regions associated with the wakes of the four rotors. As the flow moves downstream, the wakes of the individual rotors start mixing with each other, which makes the whole wake of the MR4 turbine more uniformly distributed at $x=4 D$. Further downstream at $x=7 D$, the wake of the MR4 turbine resembles the one of the SR turbine, with a maximum velocity deficit at $(y, z)$ $\simeq\left(0, z_{h}\right)$. Ultimately, the wakes of the SR and MR4 turbines have almost identical mean flow distribution at $x=10 D$.

In the following, we attempt to unravel how the MR4 wake evolves as it moves downstream. Figure 6 shows variation of the normalized wake center velocity $\bar{u}_{c} / \bar{u}_{h}$ as well as its normalized spatial derivative in the streamwise direction [i.e., $\left(D / \bar{u}_{h}\right) \partial \bar{u}_{c} / \partial x$ ]. The latter is used to indicate the local wake recovery rate for a given streamwise location. Note that for the MR4 turbine, the wake center velocity can be defined as either (i) the one downwind of the rotor center (average of four rotors), henceforth referred to as rotor center (RC), or (ii) the one downwind of the turbine center [i.e., at $(y, z)=(0$, $z_{h}$ )], henceforth referred to as turbine center (TC). The wake center velocities, based on both definitions, are shown in Fig. 6 for the MR4 case. The figure shows that initially (e.g., at $x=2 D$ ) the RC velocity for the MR4 case is slightly higher than the one of the SR. As discussed earlier, this is due to the fact that the rotors of the MR turbine are subject to local blockage effects. Up to around $x=4 D$, one can observe that the $\mathrm{RC}$ velocity recovers faster (i.e., higher values of $\left.\partial \bar{u}_{c} / \partial x\right)$ for the MR4. On the other hand, due to the expansion of rotor wakes, the effect of wake rotors is gradually seen at the turbine 


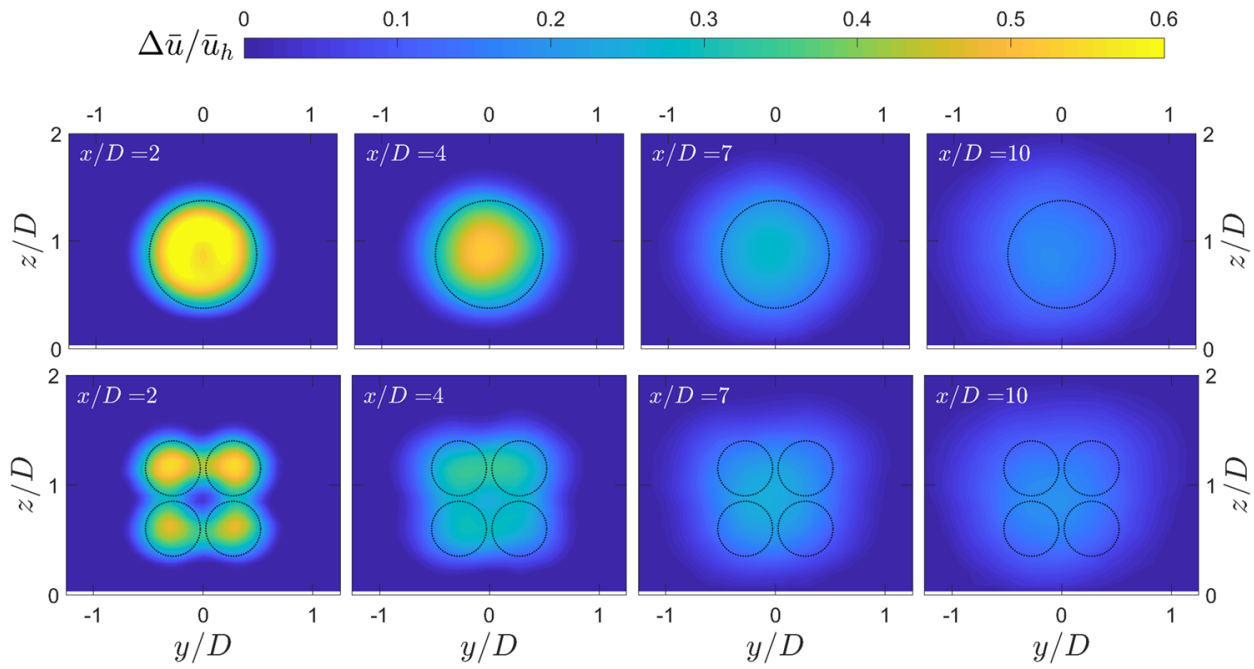

FIG. 4. Contours of the time-averaged normalized velocity deficit $\Delta \bar{u} / \bar{u}_{h}$ in vertical planes, normal to the incoming flow at different downwind locations for both the SR (top) and the MR4 turbine (bottom). For each case, the black circles show the frontal area of the wind turbine.

center (TC), and thereby the TC velocity decreases until it eventually becomes equal to the RC velocity at around $x=4.5 \mathrm{D}$. At this position, the MR4 turbine wake is expected to have a relatively uniform (i.e., top-hat) velocity distribution. One can thus observe an intermediate region ( $x=4-5 D$ for this setup), where the transition of the MR4 turbine wake from an array of wakes to one single wake occurs. Further downstream, the MR4 turbine wake behaves in a manner similar to a single turbine wake. For instance, velocity deficit profiles can be approximated by a Gaussian distribution with the maximum value at the TC. Akin to the single turbine wake, Fig. 6 also shows that the wind speed recovery rate of the TC velocity for the MR4 turbine becomes positive in the post-transition region.

In terms of wake recovery, Fig. 6 reveals that the MR4 turbine wake recovers faster than the SR turbine wake at first, where it is characterized as a wake array. Further downstream, wake rotors, however, form a single wake that recovers slower than a SR turbine wake. To better explain this process, we carry out an analysis of the mean kinetic energy budget for the wakes of both turbines. As shown in previous studies, ${ }^{31-38}$ this analysis can provide valuable insights into the physics of energy exchanges between the incoming flow and
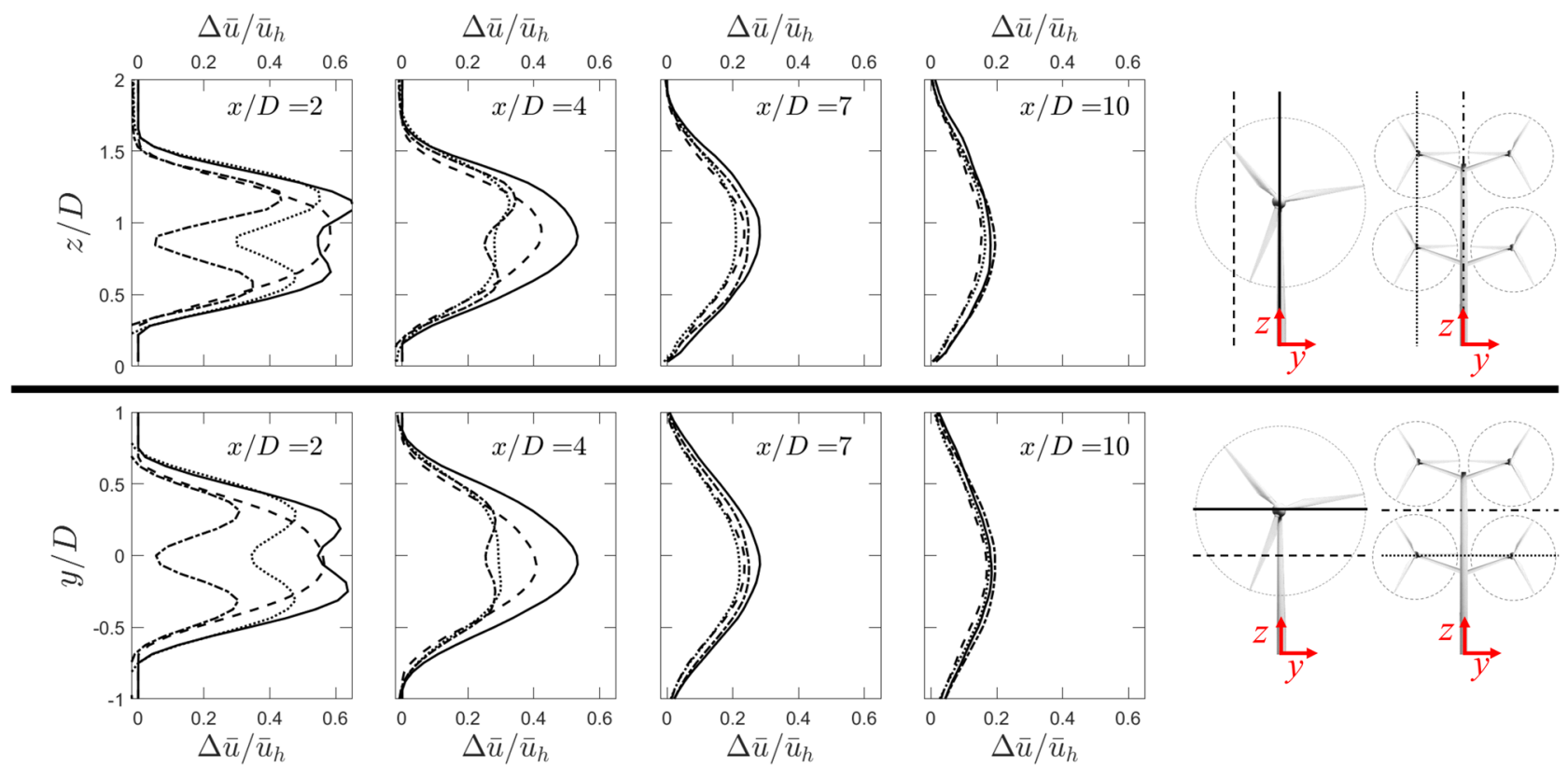

FIG. 5. Vertical (top) and lateral (bottom) profiles of the normalized velocity deficit $\Delta \bar{u} / \bar{u}_{h}$ at different downwind locations. Line plots are described by the pictorial legend shown next to the figure. 


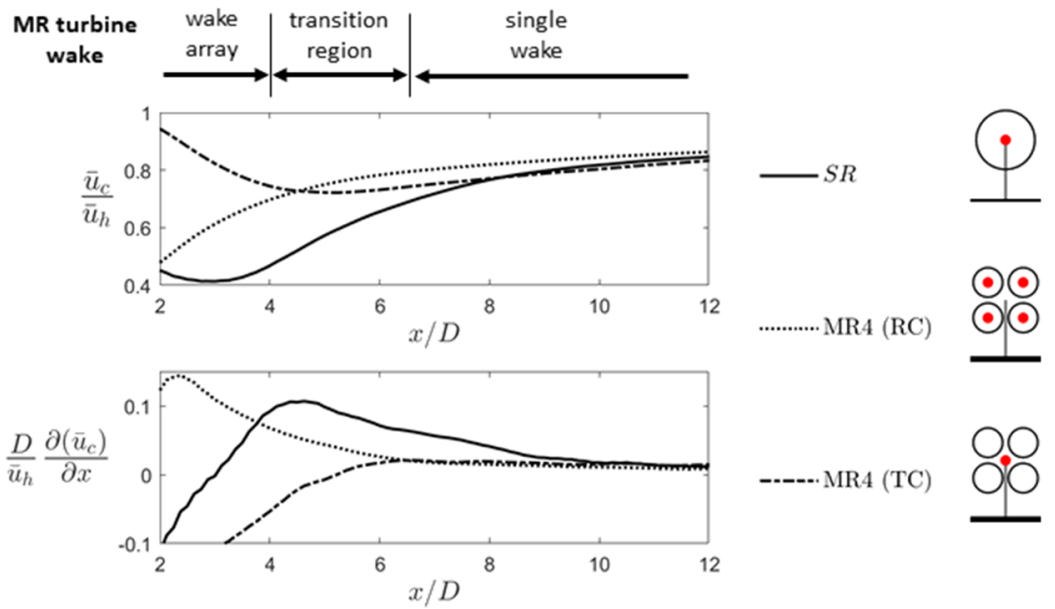

FIG. 6. Variation of the normalized streamwise wake center velocity (top) and its derivative (bottom) with the streamwise distance. For the MR4 turbine, RC denotes the streamwise velocity downwind of the rotor center (averaged of four rotors), while TC represents the velocity downwind of the turbine center with $(y, z)$ equal to $\left(0, z_{h}\right)$.

turbine wakes. The kinetic energy budget of the mean flow in the steady-state form can be mathematically expressed as ${ }^{36}$

$\underbrace{\bar{u}_{j} \frac{\partial \bar{K}}{\partial x_{j}}}_{A d v .}=\underbrace{-\frac{\bar{u}_{i}}{\rho_{o}} \frac{\partial \bar{p}}{\partial x_{i}}}_{W_{p}} \underbrace{-\frac{\partial\left[\bar{u}_{i}\left(\bar{\tau}_{i j}+\overline{\tilde{u}_{i}^{\prime} \tilde{u}_{j}^{\prime}}\right)\right]}{\partial x_{j}}}_{T_{t}}+\underbrace{\left(\bar{\tau}_{i j}+\overline{\tilde{u}_{i}^{\prime} \tilde{u}_{j}^{\prime}}\right) \frac{\partial \bar{u}_{i}}{\partial x_{j}}}_{-P_{s}}-\underbrace{\frac{\bar{u}_{i} \bar{f}_{i}}{\rho_{o}}}_{W_{t}}$,

where $\tilde{u}_{i}^{\prime}=\tilde{u}_{i}-\bar{u}_{i}$. The kinetic energy of the mean flow is denoted by $\bar{K}=0.5 \bar{u}_{i} \bar{u}_{i}$. The term denoted by $A d v$. represents the advection of the mean kinetic energy by the mean flow, $W_{p}$ shows the work done by the mean pressure gradient, $T_{t}$ describes the mean kinetic energy transport via turbulence, $P_{s}$ represents the production of turbulent kinetic energy due to flow shear, and $W_{t}$ is the work done by the turbine.

Downstream of a turbine, $W_{t}$ is zero since there are no turbineinduced forces at those locations. Hence, the advection term ( $A d v$.) in Eq. (5) is balanced by the sum of pressure, transport, and production terms in the far-wake region of a turbine.

Figure 7 shows contours of the pressure term $W_{p}$, normalized by $\bar{u}_{h}^{3} / D$, for the wakes of both turbines in vertical planes, normal to the incoming flow, at different streamwise locations. As expected, due to the recovery of air pressure behind the turbine, $W_{p}$ is mostly negative in the wake region. It will be, however, seen later that $W_{p}$ has small values compared to the transport and production terms in the far-wake region, where the change in air pressure is negligible.

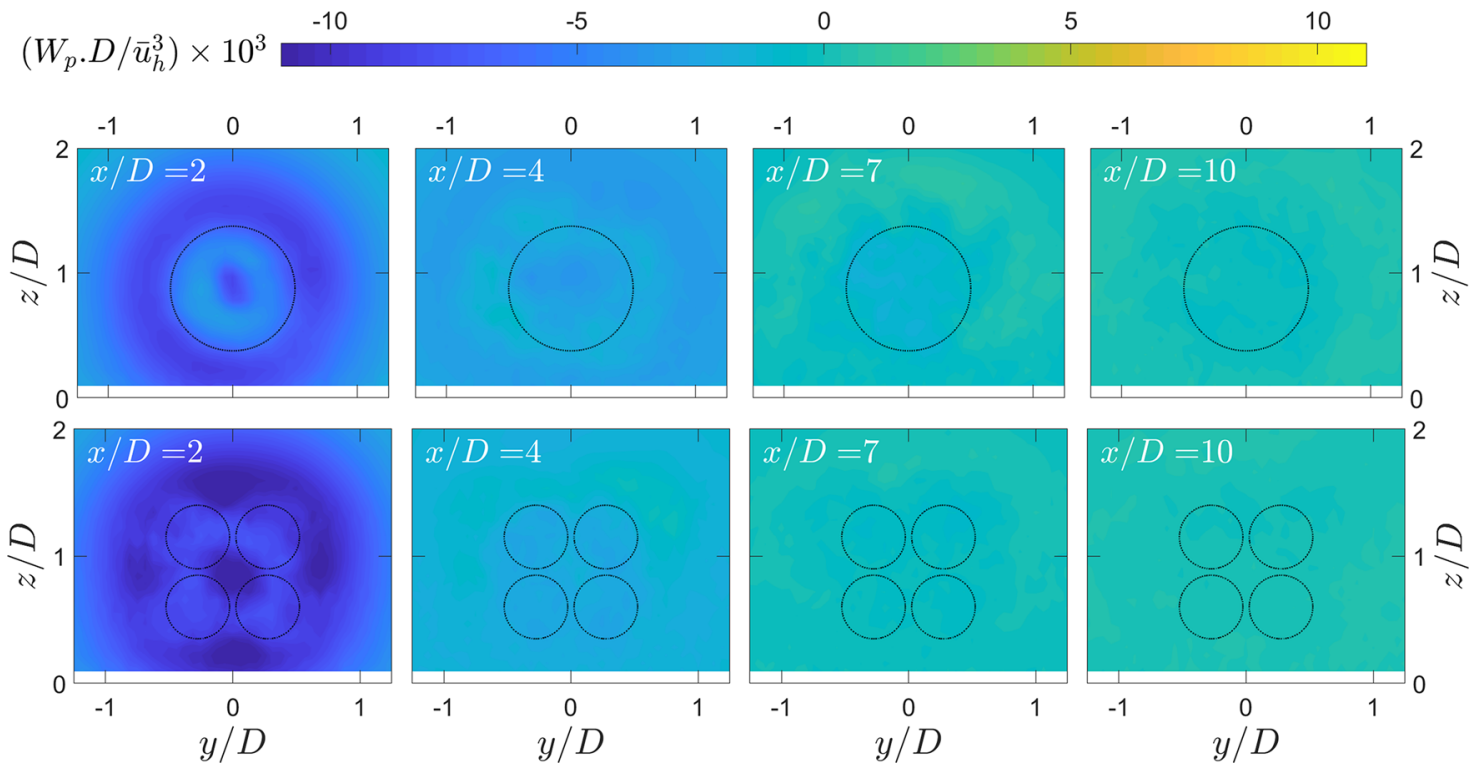

FIG. 7. Contours of the mean pressure-gradient work $W_{p}$, normalized by $\bar{u}_{h}^{3} / D$, in vertical planes, normal to the incoming flow, at different downwind locations for both the SR (top) and the MR4 turbine (bottom). For each case, the black circles show the frontal area of the wind turbine. 
Hence, the main focus will be given to the production and transport terms in the following.

Contours of the production term $P_{s}$ at different streamwise positions are shown in Fig. 8. Given that $P_{s}$ is generally positive, this term is a sink of energy in Eq. (5), and it basically shows how much energy is transferred from the mean flow to turbulence. As seen in the figure, $P_{s}$ has generally high values at the outer edges of wakes, which is associated with the strong mean flow shear at these locations. ${ }^{18}$ In addition, due to the strong flow shear at the center of the MR4 wake at $x=2 D$ (see Fig. 5), Fig. 8 shows that there is a considerable amount of turbulence production in this region. Further downstream, however, the MR4 wake forms a single wake with a more uniform distribution as discussed earlier. This diminishes the turbulence production in the MR4 wake particularly at the wake center for $x \geq 4 D$. In comparison with the SR wake, there is also lower turbulence production at the edges of the MR4 wake at this streamwise region. This is expected given that overall the MR4 wake has a more uniform velocity distribution, as shown in Fig. 5, compared to the SR turbine wake.

As we know, turbulence plays a key role in transporting the mean kinetic energy from one location to another in turbulent flows. The amount of this energy transport is quantified by the transport term $T_{t}$ in Eq. (5). Contours of the normalized $T_{t}$ are shown in Fig. 9 for the wakes of two turbines at different streamwise positions. One can observe that $T_{t}$ is generally negative for the outer flow and positive within the wake. Moreover, the magnitude of $T_{t}$ is overall larger than $P_{s}$. This suggests that although some amount of mean kinetic energy is consumed by $P_{s}$ to generate turbulence, the generated turbulence has a more profound effect on the mean kinetic energy by transferring the energy from outer flow into the wake. This phenomenon is often called energy entrainment which occurs for any turbulent wake flow. ${ }^{39}$
For the MR4 wake at $x=2 D$, the energy transport from surroundings seems to be more effective. This can be explained by (i) higher values of $P_{s}$ at the edges of the MR4 wake and also (ii) larger wake boundaries (i.e., wake perimeter) for the MR4 wake. In addition to the energy entrainment from surroundings, at $x=2 D$, there is another strong energy entrainment from the MR4 wake center, as shown in Fig. 9. The central part of the MR4 wake at $x=2 D$ is characterized by relatively high levels of both wind speed (Fig. 4) and generated turbulence (Fig. 8), both of which enhance the energy transport $T_{t}$ according to Eq. (5). This explains why the MR4 wake initially recovers faster, as shown in Fig. 6. The transition of the MR4 wake from an array of wakes to a single wake that occurs further downstream, however, terminates the energy transport from the central part. Lack of energy entrainment from the center together with lower entrainment from surroundings (due to the lower level of turbulence at wake edges) makes the MR4 wake recover slower than the SR one in the post-transition region.

Despite the favorable impact of generated turbulence on energy entrainment and wake recovery, unsteadiness caused by turbulence increases fatigue loads on downwind wind turbines. ${ }^{40}$ To shed light on this problem, we conclude this section by comparing the amount of wake turbulence added by the MR4 turbine with the one corresponding to the SR turbine wake. Streamwise turbulence intensity at a given location added due to the presence of the turbine is given by

$$
\begin{aligned}
I^{+}=+\sqrt{I^{2}-I_{\text {in }}^{2}} & I \geq I_{\text {in }}, \\
I^{+}=-\sqrt{I_{\text {in }}^{2}-I^{2}} & I<I_{\text {in }},
\end{aligned}
$$

where $I_{\text {in }}$ is the incoming streamwise turbulence intensity plotted in Fig. 1. Figure 10 shows contours of added streamwise turbulence intensity $\mathrm{I}^{+}$in vertical planes normal to the incoming flow for both

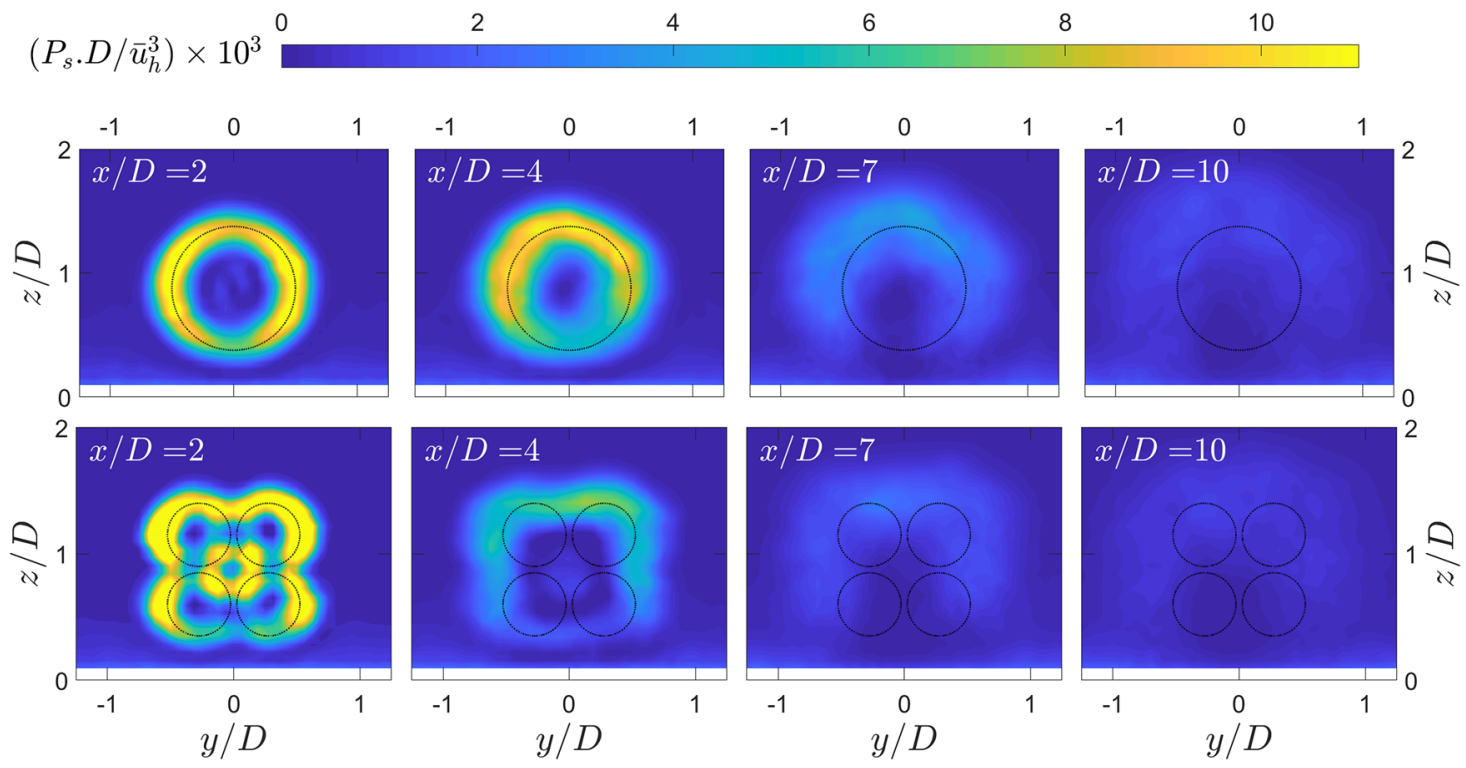

FIG. 8. Contours of the turbulence production $P_{s}$ due to flow shear, normalized by $\bar{u}_{h}^{3} / D$, in vertical planes, normal to the incoming flow, at different downwind locations for both the SR (top) and the MR4 turbine (bottom). For each case, the black circles show the frontal area of the wind turbine. 


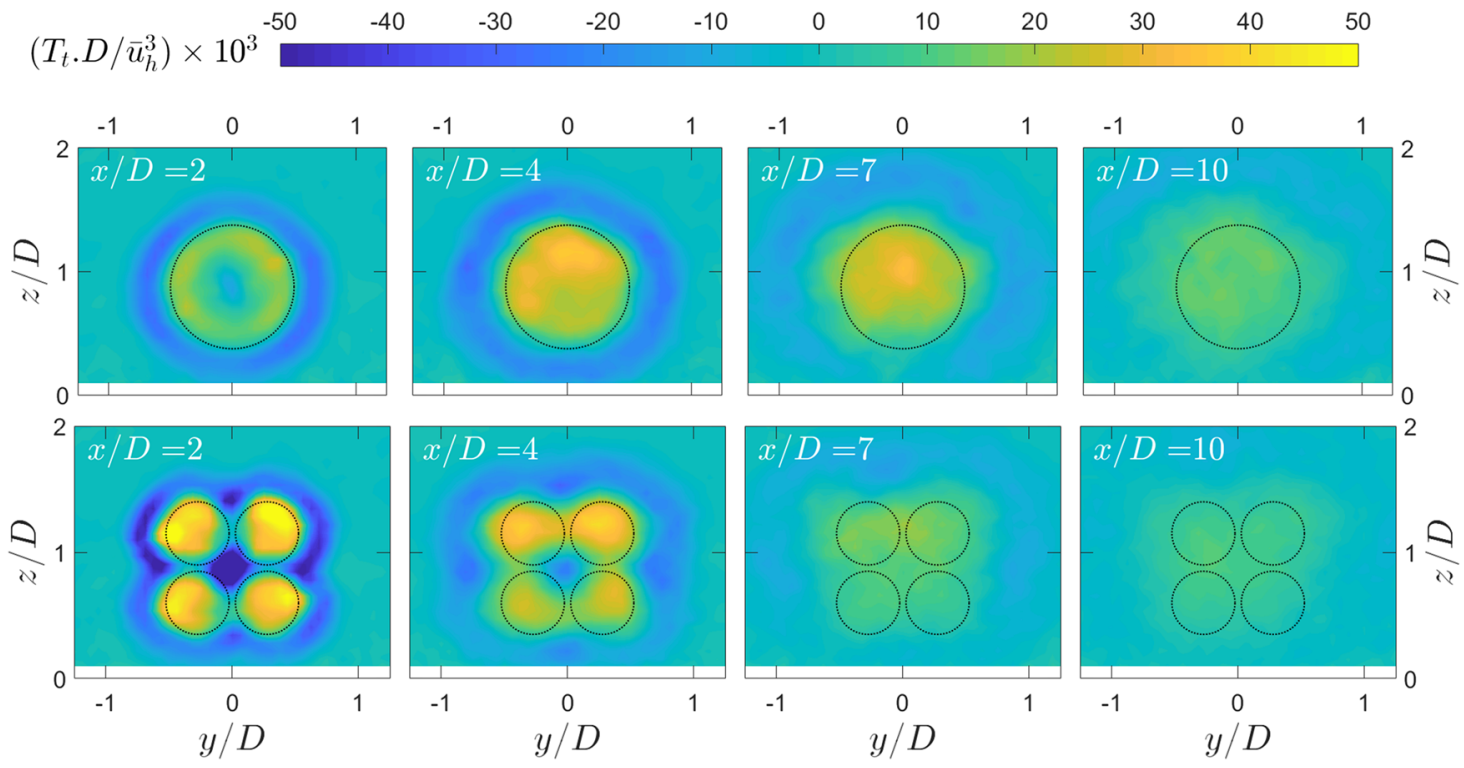

FIG. 9. Contours of mean kinetic energy transport $T_{t}$ via turbulence, normalized by $\bar{u}_{h}^{3} / D$, in vertical planes, normal to the incoming flow, at different downwind locations for both the SR (top) and the MR4 turbine (bottom). For each case, the black circles show the frontal area of the wind turbine.

turbines at several downwind locations. Vertical and lateral profiles of $I^{+}$are also shown in Fig. 11. In general, we observe that although the turbulence added by the MR4 turbine is higher for smaller downwind distances, it becomes lower than the one of the SR turbine at $x>4$. Given the fact that the turbine spacing in wind farms is usually equal or bigger than $5 D$, this highlights another advantage of using
MR turbines in wind farms. Additionally, even though both turbines suppress turbulence in the proximity of ground at large downwind distances, the MR turbine does it more profoundly. The suppression of turbulence in this region is most likely due to the reduction in the mean shear and associated turbulence production (shown in Fig. 8) caused by the presence of the turbine. ${ }^{41-43}$

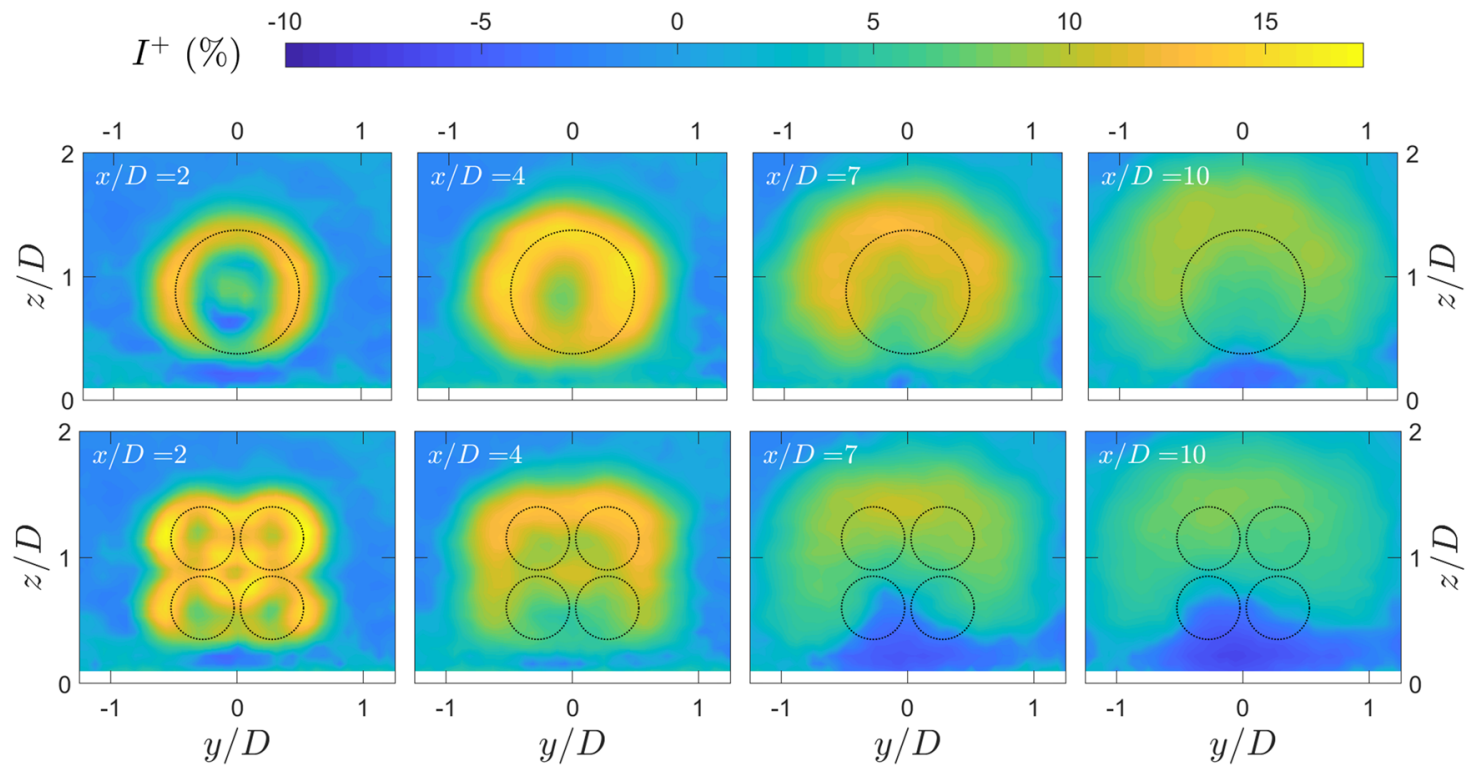

FIG. 10. Contours of added streamwise turbulence intensity $I^{+}$in vertical planes, normal to the incoming flow, at different downwind locations for both the SR (top) and the MR turbine (bottom). For each case, the black circles show the frontal area of the wind turbine. 


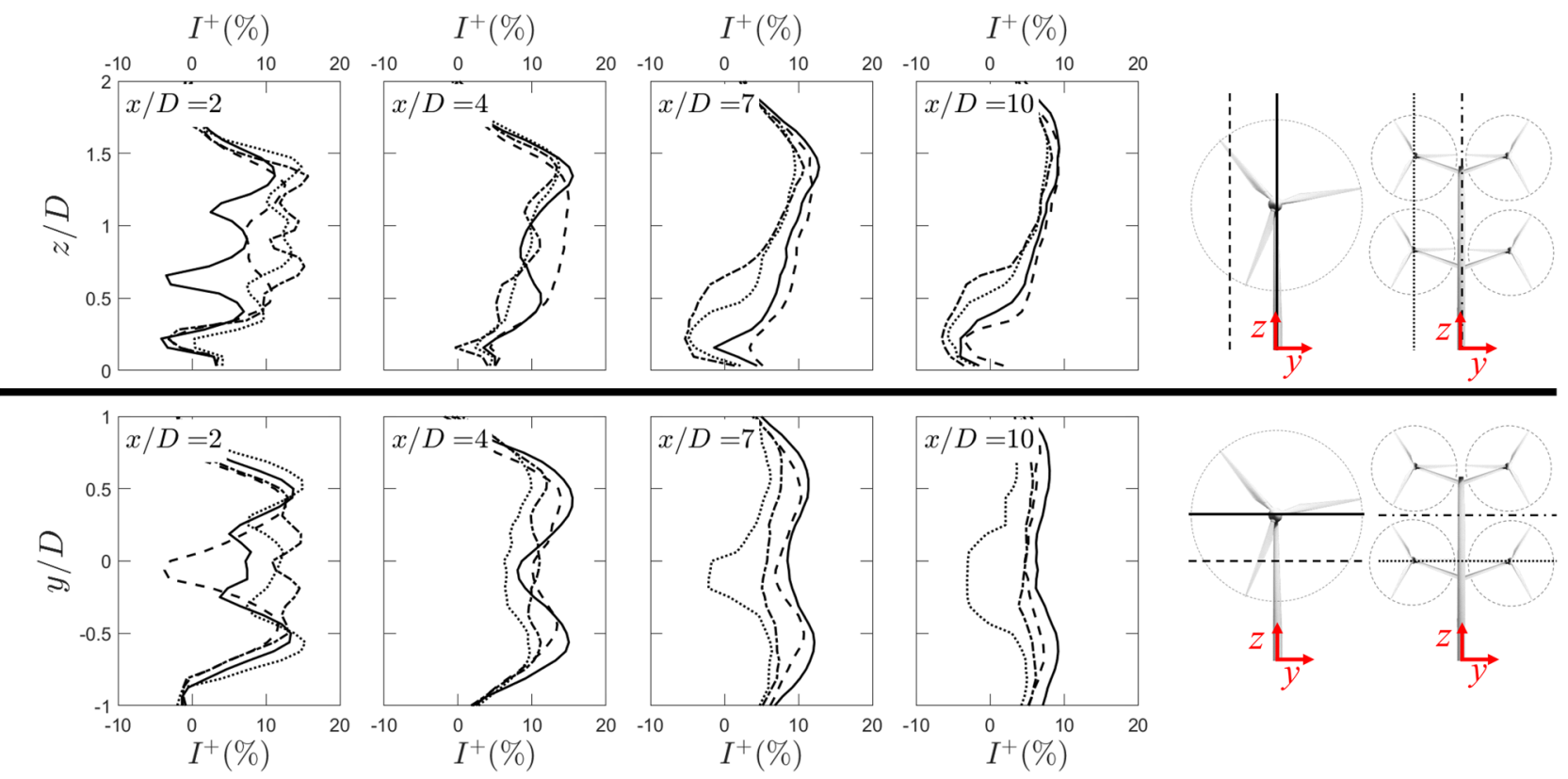

FIG. 11. Vertical (top) and lateral (bottom) profiles of added streamwise turbulence intensity $I^{+}$at a few downwind locations. Line plots are described by the pictorial legend shown next to the figure.

\section{B. Multirotor turbine wakes: Effect of rotor spacing}

Spacing between SR wind turbines within wind farms is known to have a substantial impact on wake flow distributions. ${ }^{44,45}$ For a MR turbine, Ghaisas et al. ${ }^{12}$ studied the effect of rotor spacing on the mean wake flow downwind of the turbine. They reported that the disk-averaged velocity in the wake region is relatively insensitive to the rotor spacing. However, they considered a relatively large tip clearance (factors of $1 d$ ) in their simulations. Given structural constraints, the tip clearance of MR turbines is expected to be considerably smaller than $1 d$, therefore, it is of interest to examine the impact of more realistic rotor spacing values on MR turbine wakes. Figure 12 shows the variation of $\langle\Delta \bar{u}\rangle / \bar{u}_{h}$ in the streamwise direction for the wake of the SR turbine as well as the MR4 one with three different values of rotor spacing (i.e., $s=0,0.1 d, 0.25 d$ ). It can be seen that, with respect to the SR turbine, the MR4 turbine has low values of velocity deficit in the streamwise range of $2-8 D$, where a downwind turbine is most likely located, for all the tested rotor spacing. Moreover, the figure shows that an increase in rotor spacing further reduces the wake velocity deficit. This can be explained by considering the fact that an increase in rotor spacing extends the streamwise distance that each rotor wake can travel downstream without having strong interactions with the wake of adjacent rotors. In other words, an increase in rotor spacing postpones the transition from a wake array to a single wake. To better examine the effect of spacing on the wake transition, we define the streamwise transition length $L_{T}$ as the streamwise distance at which the velocity downwind of the turbine center (TC) becomes equal to the one downwind of the rotor center (RC) (average of four rotors). Figure 13(a) shows the value of $L_{T}$ based on the LES results for the three different values of rotor spacing. We observe that $L_{T}$ increases approximately linearly with the rotor spacing $s / d$. Analytical wake modeling can be used to predict the transition length $L_{T}$. In general, an analytical wake model describes the wake velocity 

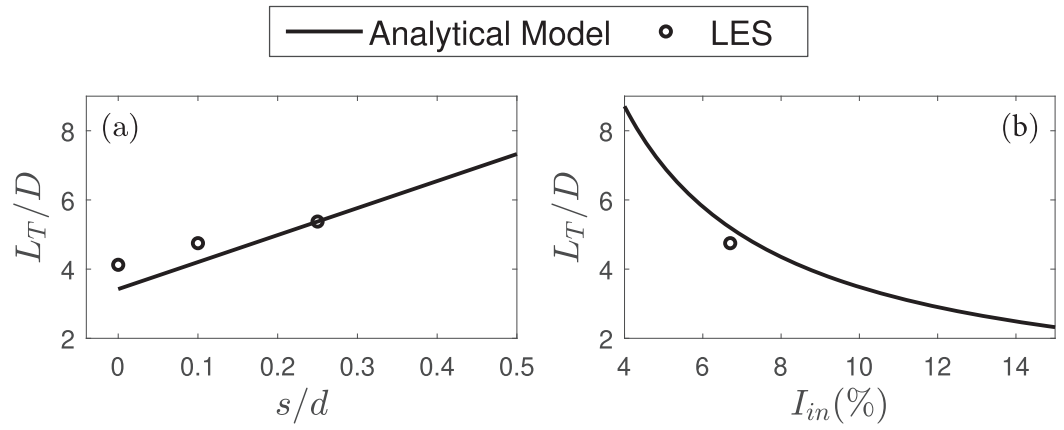

FIG. 13. The normalized transition length $L_{T} / D$ of the MR4 turbine wake for different values of (a) rotor spacing s/d and (b) incoming turbulence intensity $I_{\text {in }}(\%)$. For the latter, $s$ is equal to $0.1 d$. deficit as

$$
\frac{\Delta \bar{u}}{\bar{u}_{h}}=C(x) f(r)
$$

where $C$ is the maximum velocity deficit for a given streamwise location. $f(r)$ represents the velocity deficit distribution as a function $r$, where $r$ is the radial distance from the rotor center. For instance, $f(r)$ is assumed to be a Gaussian distribution in the model developed by Bastankhah and Porté-Agel. ${ }^{46}$ In order to find $L_{T}$, we next use Eq. (7) to calculate the velocity deficit at the turbine center (TC) as well as the rotor center (RC). Note that linear superposition of velocity deficit is used to account for the velocity deficit caused by all the four rotors. The validity of linear superposition for MR turbine wakes is later elaborated in Sec. III D. At a given streamwise location, the resultant velocity deficit at the turbine center $\Delta \bar{u}_{T C}$ can be written as

$$
\frac{\Delta \bar{u}_{T C}}{\bar{u}_{h}}=4 C(x) f\left(\frac{d+s}{\sqrt{2}}\right) .
$$

Note that the radial distance between each rotor center and the turbine center is $(d+s) / \sqrt{2}$ (see Fig. 2). Likewise, the resultant velocity deficit behind a rotor center $\Delta \bar{u}_{R C}$ is equal to

$$
\frac{\Delta \bar{u}_{R C}}{\bar{u}_{h}}=C(x)+2 C(x) f(d+s)+C(x) f(\sqrt{2}(d+s)) .
$$

The second and third terms on the right side of Eq. (9) indicate the velocity deficit caused by the adjacent rotors. At $x$ equal to $L_{T}$, $\Delta \bar{u}_{T C}$ given by Eq. (8) should be the same as $\Delta \bar{u}_{R C}$ determined by Eq. (9). Equating Eqs. (8) and (9), removing $C(x)$ from both sides, and substituting $(s+d) / \sqrt{(2)}$ with $r^{*}$ yield

$$
1+2 f\left(\sqrt{2} r^{*}\right)+f\left(2 r^{*}\right)=4 f\left(r^{*}\right) .
$$

If $f(r)$ is expressed as a Gaussian profile, $f(a r)$ is equal to $f^{\left(a^{2}\right)}(r)$. Therefore, Eq. (10) can be rewritten as

$$
f^{4}\left(r^{*}\right)+2 f^{2}\left(r^{*}\right)-4 f\left(r^{*}\right)+1=0 .
$$

Solving the above polynomial equation to find its real roots, we get either $f=1$ or $f \approx 0.2956$. The Gaussian distribution of $f\left(r^{*}\right)$ can be mathematically expressed as

$$
f\left(r^{*}\right)=\exp \left(-\frac{r^{* 2}}{2 \sigma^{2}}\right)
$$

where $\sigma$ is the wake width at a given streamwise location. Based on Eq. (12), $f=0$ is satisfied only at $x \rightarrow \infty$, where $\sigma$ is infinitely large, and therefore at $x=L_{T}, f \approx 0.2956 . \sigma$ at $x=L_{T}$ is given by ${ }^{46}$

$$
\frac{\sigma}{d}=k^{*} \frac{L_{T}}{d}+\epsilon
$$

where $k^{*}$ is the wake growth rate and $\epsilon$ is the normalized initial wake width, which is a function of the turbine thrust coefficient (see the original paper ${ }^{46}$ for more information). From Eqs. (12) and (13) and bearing in mind that $f \approx 0.2956$ and $r^{*}=(d+s) / \sqrt{2}$, one can conclude that

$$
\frac{L_{T}}{d} \approx \frac{1}{k^{*}}\left[\frac{1+(s / d)}{2.2}-\epsilon\right]
$$

The solid line in Fig. 13 shows the predictions of $L_{T}$ based on the above equation. The wake growth rate $k^{*}$ is estimated based on the empirical relationship suggested in Ref. 47 . Note that, for being consistent with the rest of the manuscript, $L_{t}$ is normalized by $D$ in Fig. 13. Therefore, the shown values for $L_{t}$ in the figure are half of those calculated by Eq. (14). The figure shows that this simple relationship is able to rather acceptably predict the transition length for MR turbine wakes with different values of rotor spacing. Moreover, it can be used to examine the effect of other parameters on the wake transition length. For instance, Fig. 13(b) shows the variation of $L_{T}$ with the incoming turbulence for a rotor spacing of $s=0.1 d$. It can be seen that the transition to a single wake occurs much faster for more turbulent inflow conditions. For instance, $L_{T}$ is only slightly more than $2 D$ for $I_{\text {in }}$ equal to $15 \%$. This suggests that the use of MR turbines in wind farms is more likely to be profitable if they operate in smooth inflows such as those at offshore locations. Figure 13(b) can also explain recent results reported by van der Laan and Abkar ${ }^{48}$ for an array of MR turbines. They found that the wind farm efficiency can be improved by using MR turbines instead of SR turbines. However, this improvement is only limited to a few first turbine rows in a wind farm, and the performance of MR turbines further downstream is similar to those in a wind farm consisting of SR turbines. This observation can be explained by the fact that a higher level of turbulence within wind farms leads to an earlier transition of MR turbine wakes to single wakes, as shown in Fig. 13(b). As a result, wakes of MR turbines deep inside wind farms do not recover considerably faster than those of SR turbines.

It is important to note that our LES results are not sufficient to validate the analytical relationship discussed above, especially for the effect of incoming turbulence intensity, and more rigorous 
validation studies need to be done in future works. It might also be worth recalling that Eq. (14) is derived for a MR4 turbine with the geometrical layout shown in Fig. 2. Similar relationships can be, however, derived for MR turbines with other geometrical layouts.

We conclude this section by pointing out that our results show that the MR4 turbine power is about 2\% more than that of the SR turbine, which is in agreement with findings of van der Laan et al. ${ }^{1 .}$ Note that the reported power increase for the MR4 turbine is found to be relatively the same for the three studied values of rotor spacing.

\section{Wake recovery: Effect of rotor size}

The budget analysis of mean kinetic energy showed that MR turbines benefit from a faster wake recovery at short and moderate downwind distances as they are characterized by higher turbulence production due to higher mean flow shear (i.e., velocity gradients). Among different velocity gradient terms contributing to a higher shear production, $\partial \bar{u} / \partial y$ and $\partial \bar{u} / \partial z$ are arguably the most dominant ones in the far-wake region. These two terms are in the same order of magnitude as $\Delta \bar{u}_{R C} / l$, where $l$ is the cross-stream length scale. Assuming the wake expands linearly, $l$ is proportional to the rotor diameter. ${ }^{49}$ Therefore, one can relate the initial faster wake recovery observed for MR turbines to their smaller rotor size since a smaller rotor size leads to a shorter cross-stream length scale ${ }^{12}$ and, thereby, stronger values of velocity gradients (i.e., $\Delta \bar{u}_{R C} / l$ ).

To better understand how the wake recovery is influenced by the rotor size, LESs are performed to compare wakes of SR $n$ turbines with the same hub height but with different rotor diameters, denoted by $D_{n}=D / \sqrt{n}$, where $n$ varies from 1 to 7 . Figure 14(a) shows the variation of the normalized maximum velocity deficit with the streamwise distance, normalized by $D$. The figure clearly shows that the smaller the turbine is, the faster its wake recovers. This is indeed due to the fact that a given streamwise location is in fact much further downstream for a smaller turbine, with respect to its rotor size. For instance, a streamwise distance of $4 D$ is equivalent to $8 D_{4}$ for a SR4 turbine. If the streamwise distance for each turbine is normalized by its corresponding rotor diameter $D_{n}$, then Fig. 14(b) shows that all the curves approximately collapse onto a single curve representing a universal behavior of turbine wakes in a coordinate system that is normalized by each turbine rotor diameter. This can also be verified by the expression used to quantify wake growth in analytical wake models. For instance, as discussed in Sec. III B, the wake width $\sigma$ for a SR $n$ turbine can be estimated by $k^{*} x / D_{n}+\epsilon$. Therefore, the wake recovery rate in global coordinates is, in fact, $k^{*} / D_{n}$, which indeed increases with the decrease in $D_{n}$. For a coordinate system normalized by $D_{n}$, however, the wake recovery rate is equal to $k^{*}$. The value of $k^{*}$ is known to mainly depend on incoming flow conditions, which are almost the same for the different SR turbines as they are subject to the same boundary-layer inflow. Note that Fig. 14(b) shows a slight difference in the wake velocity deficit profiles associated with different turbines. This discrepancy might be due to the fact that rotors with different sizes are subject to slightly different incoming velocity profiles, given the logarithmic shape of the boundary-layer flow. This can be also partly due to different levels of turbulence generated by flow shears in wakes of rotors with different sizes.

Bearing the results shown in this section in mind, it is of interest to investigate the wakes of MR turbines with different sizes of rotors. This is discussed in Sec. III D.

\section{Multirotor turbine wakes: Effect of rotor number}

In this section, we examine the effect of numbers of rotors on the wake of a MR turbine. In addition to the MR4 turbine, LESs are performed to quantify flow distributions downwind of a MR2 turbine and a MR7 one. Variations of the normalized velocity deficit, averaged over rotors, with streamwise distance are plotted in Fig. 15 for different turbines. For the sake of a fair comparison, the same normalized spacing (i.e., $s=0.1 d$ ) is used for all of the MR turbines. The figure shows that the increase in the number of rotors from two to four leads to the reduction in the velocity deficit in the wake at moderate downwind distances (i.e., $x<5 D$ ). However, a further increase in rotor numbers to seven results in a marginal reduction in the wake velocity deficit.

Even though, based on Fig. 14, wakes of smaller single rotors recover faster, Fig. 15 shows that the faster recovery of a single wake does not necessarily lead to an overall faster wake recovery when there is an array of rotors operating as a MR turbine (e.g., compare
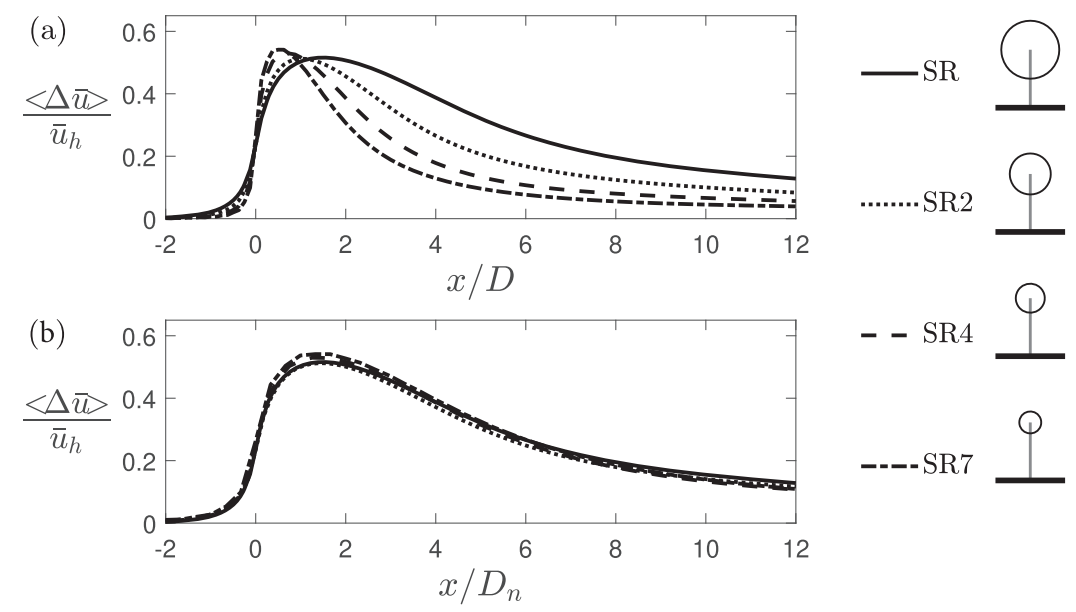

FIG. 14. Variation of the normalized maximum velocity deficit (a) with streamwise distance normalized by $D$ and (b) with streamwise distance normalized by $D_{n}$. The value of $D_{n}$ for a SRn turbine is equal to $D / \sqrt{n}$.

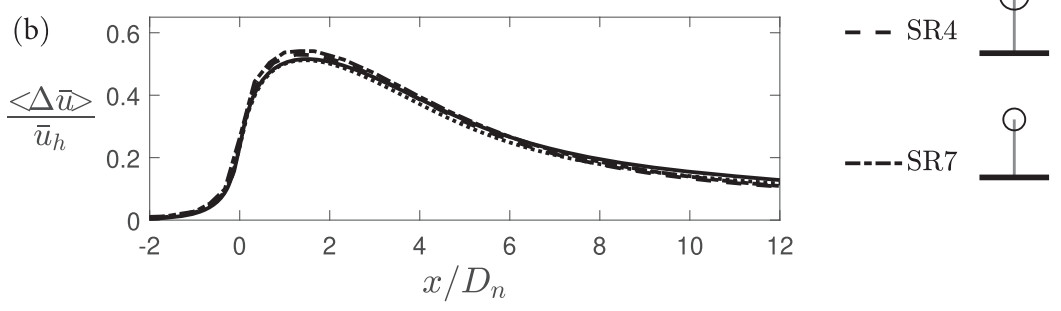




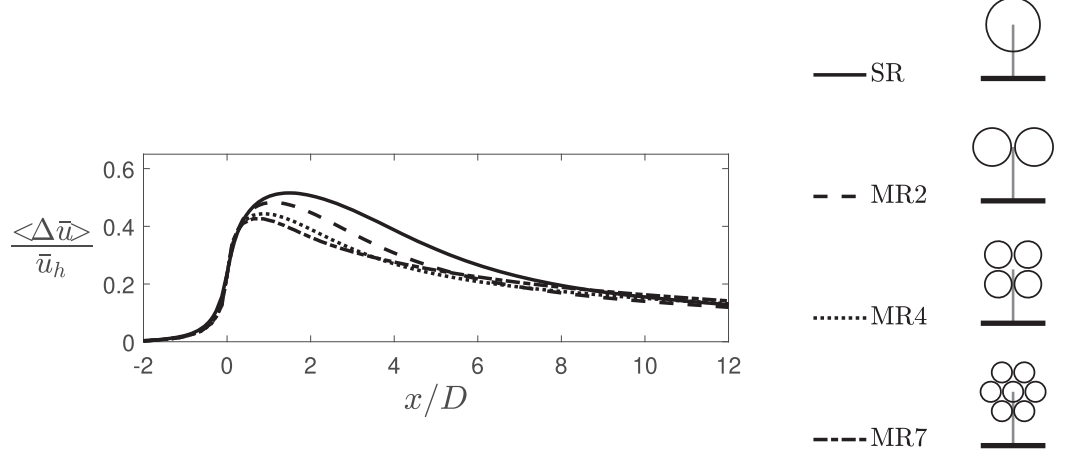

FIG. 15. Variation of the normalized velocity deficit, averaged over rotors, with streamwise distance for turbines with different numbers of rotors. the wake of a MR7 turbine with the one of MR4 turbine). To better understand the reason behind this, it can be useful to look at Fig. 16, which shows the lateral profiles of the normalized velocity deficit for different turbines. As shown on schematic turbines depicted on the right side of the figure, the profiles are plotted at the hub height for all of the MR turbines, except for the MR4 turbine. For this turbine, lateral profiles are plotted at the height where the wake is initially stronger [i.e., $z=z_{h}+(d+s) / 2$ ]. The figure clearly shows that wakes of MR turbines with higher numbers of rotors have lower values of velocity deficit at first (e.g., $x=2 D$ ). As shown in Fig. 14, this is mainly due to the faster wake recovery for smaller rotors. However, the figure shows that the transition from a wake array to a single wake occurs much faster for MR turbines with higher numbers of rotors. This is because the use of a higher number of rotors makes the whole wake flow distribution more uniform. For instance, based on Fig. 16, one can infer that $L_{T}$ is about $7 D$ for the MR2 turbine, while it is reduced to about $4 D$ for a MR4 turbine, and it is indeed less than $4 D$ for the MR7 turbine. For streamwise distances greater than $L_{T}$, the cross-stream length scale is proportional to $D$ rather than $d$, which results in a much slower wake recovery. In other words, for a given MR turbine with high number of rotors, the early transition to a single wake can overshadow the initial fast recovery of small rotor wakes.

To predict wake flow distributions for MR turbines with higher numbers of rotors, the Gaussian wake model proposed by Bastankhah and Porté-Agel ${ }^{46}$ is employed. As mentioned earlier, the wake recovery rate $k^{*}$ for the analytical wake model is estimated based on the empirical relationship reported by Ref. 47 . To estimate the whole flow distribution in a MR turbine wake, the wake of rotors can be superposed either linearly or nonlinearly. Based on the linear superposition of rotor wakes, the velocity deficit $\Delta \bar{u}$ for a MR turbine wake is given by $\sum_{i=1}^{n} \Delta \bar{u}_{i}$, where $\Delta \bar{u}_{i}$ is the velocity deficit caused by the $i$ th rotor. Alternatively, based on the nonlinear superposition method, the total velocity deficit $\Delta \bar{u}$ is estimated by $\sqrt{\sum_{i=1}^{n}\left(\Delta \bar{u}_{i}\right)^{2}}$. Predictions of the Gaussian wake model using both of these superposition methods are shown in Fig. 16. The figure reveals that the linear superposition provides more satisfactory predictions, which is in agreement with the results of Ghaisas et al. ${ }^{12}$ The results of the two different superposition methods deviate more from each other for MR turbines with higher numbers of rotors. According to the analytical model predictions, in the far-wake region, wakes of MR19 and MR37 have values of velocity deficit relatively similar to those of other MR turbines, despite having lower velocity deficit values at first. Note that, however, analytical model predictions for MR turbines with high numbers of rotors at long downwind distances (e.g., 10D) should be treated with extra care since the validity of some assumptions used to develop the Gaussian wake model (e.g., linear growth of the rotor wakes) can be questionable very far from each rotor. Note that, for instance, $x=10 D$ is about $44 d$ and $61 d$ for MR19 and MR37, respectively.

Finally, it is worth mentioning that the LES results show that the power output of the MR wind turbines slightly increases by increasing the number of rotors. In particular, we found that the power production of the MR2, MR4, and MR7 turbines is higher than the SR turbine by about $1.5 \%, 2 \%$, and $4 \%$, respectively.

\section{E. Effect of rotation}

In Secs. III A-III D, wakes of MR turbines with conventional rotors turning clockwise, seen from upstream, are discussed. However, rotors of an MR turbine can essentially rotate in a different direction with respect to adjacent rotors. It is therefore of interest to examine the possibility of speeding up the wake recovery with the right choice of rotor rotation direction. This is based on the assumption that swirl motions induced by rotation of rotors can constructively interact with each other in such a way that they might be able to channel the outer flow into the wake and thereby enhancing flow entrainment. This hypothesis is examined in the following for an MR4 turbine with a rotor spacing $s$ of $0.1 d$. An MR4 turbine can basically operate with 16 (i.e., $2^{4}$ ) different distributions of the rotation direction. Among those, six cases that are deemed to be the most relevant ones were numerically simulated via LES, as shown in Fig. 17. Rotation directions of rotors for each case are shown schematically at the top of the figure. The figure shows contours of normalized velocity deficit overlaid with vectors of cross-stream velocity components at $x=3 D$ for different rotation cases. $R 1$ denotes the reference case at which all of the rotors turn clockwise, whereas in other five cases (R2-6), two out of four rotors turn clockwise and the other two turn counterclockwise. It is also worth mentioning that the rotation directions of rotors are seen from upstream in the schematic panel (positive $y$-axis points left), while velocity contours are plotted in a conventional viewing angle from downstream (positive $y$-axis points right). That is why it seems at first glance that rotors turn in the 


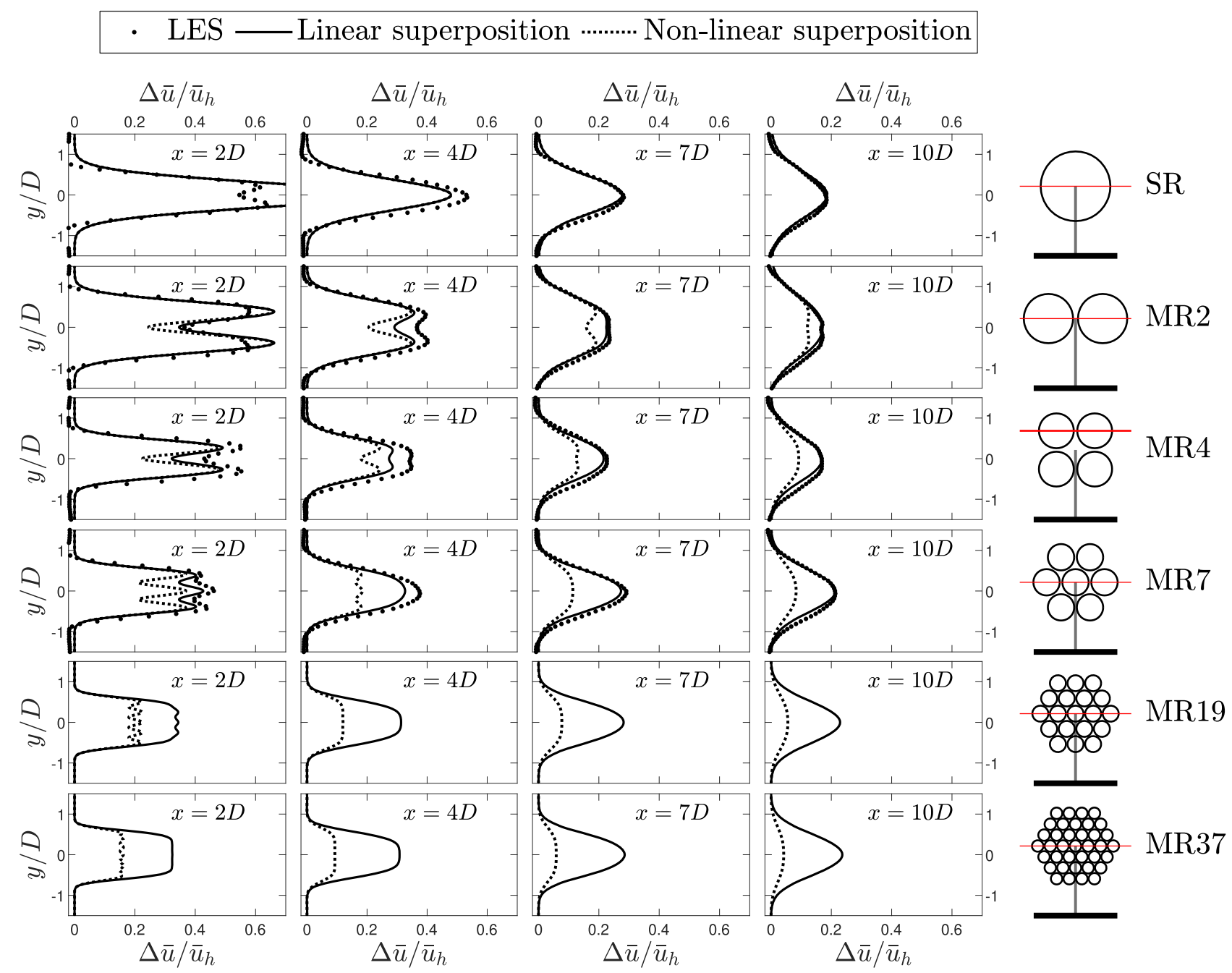

FIG. 16. Lateral profiles of the normalized velocity deficit at different streamwise locations. Each column of subfigures corresponds to a given streamwise location, while each row represents the data associated with a given turbine shown on the right side of the figure. LES data are shown by blue circles, and analytical model predictions based on linear and nonlinear superposition are shown by solid and dashed lines, respectively. As shown by solid blue lines on schematics of turbines, lateral velocity deficit profiles are plotted at $z=z_{h}$ for all of the turbines, except for the MR4 turbine for which $z=z_{h}+(d+s) / 2$.

same direction as their wakes. However, as we know, a rotor wake turns in the opposite direction of that of the rotor, according to the conservation of angular momentum. ${ }^{5}$

As observed in Fig. 17, swirl motions induced by any two adjacent rotors turning in the same direction (e.g., top rotors in $R 1$ and $R 2$ ) do not lead to significant inward momentum flux through their middle gap. It is simply due to the fact that wake rotation of one negates effects of the other. In contrast, when the two adjacent rotors turn in opposite directions with respect to each other, they both generate unidirectional momentum flux in their middle gap (e.g., top rotors in $R 3$ ). This observation suggests that cases with rotors turning in the same direction (e.g., R1) cannot contribute greatly to flow entrainment. On the other hand, for MR turbines with opposing rotation directions of rotors such as those in $R 5$ and $R 6$, it is impossible to have inward flows from all around the turbine. For instance, in $R 5$, there is lateral inward momentum flux from right and left, while the wake loses its momentum due to the vertical outward flux from above and below. Case R6 is opposite, with vertical inward momentum flux from above and below, and outward momentum flux from lateral sides.

One can infer that wakes of cases with downward momentum flux from the top (e.g., $R 4$ and $R 6$ ) are likely to recover faster given that more energy is available at higher heights of the boundary layer. To test this assumption, Fig. 18 shows variations of the spatially averaged normalized velocity deficit $\Delta\langle\bar{u}\rangle / \bar{u}_{h}$ as well as the crossstream velocity magnitude $\left\langle\sqrt{\bar{v}^{2}+\bar{w}^{2}}\right\rangle / \bar{u}_{h}$ for six different cases. As seen in the figure, the rotation direction of rotors surprisingly seems to have a negligible effect on the wake recovery. In order to better 

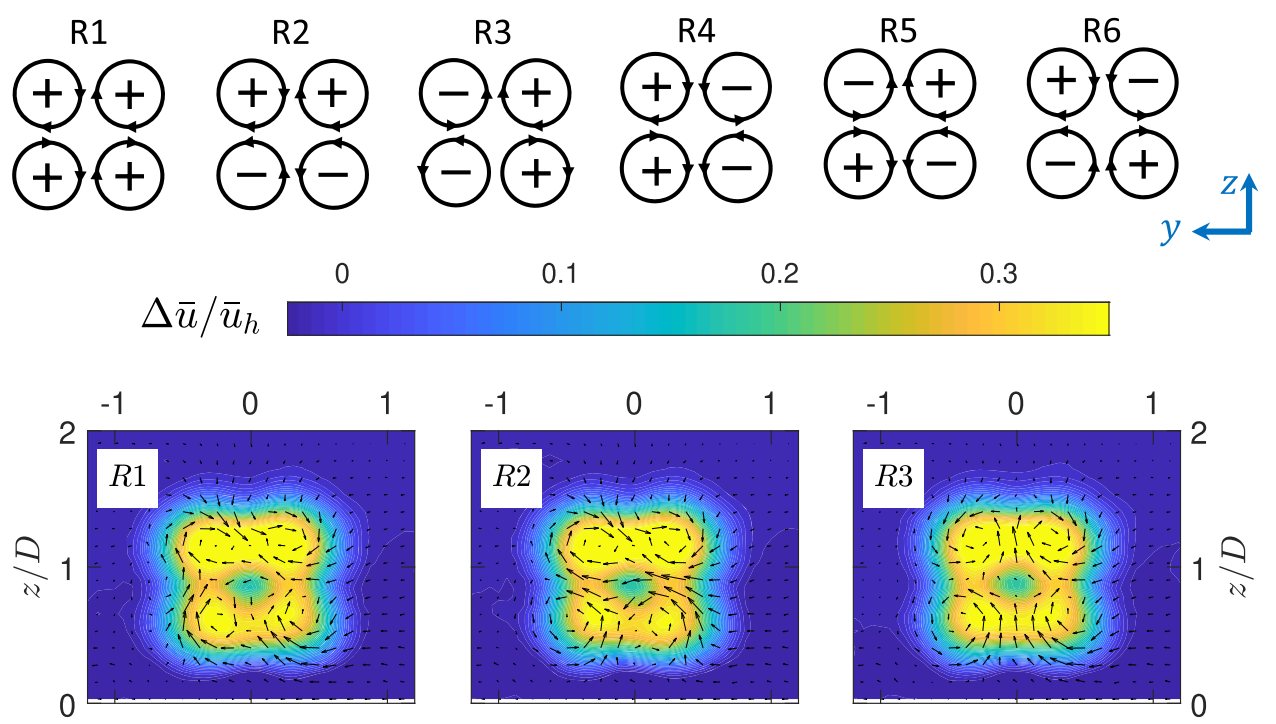

FIG. 17. Contours of the normalized velocity deficit $\Delta \bar{u} / \bar{u}_{h}$ at $x=3 D$ for an MR4 turbine $(s=0.1 d)$ with different rotation directions.
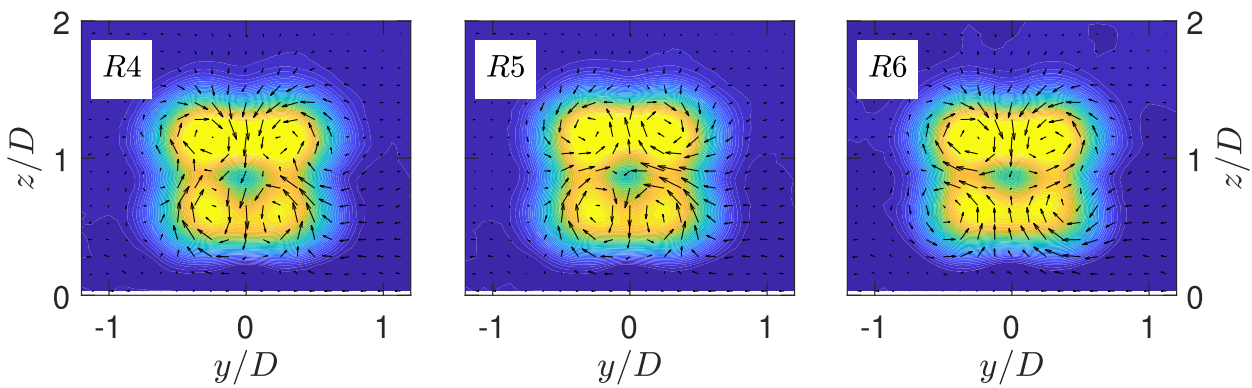

understand why cross-stream momentum fluxes shown in Fig. 17 do not impact the wake recovery, Fig. 19 shows a few representative streamlines for one of the cases, namely, $R 6$, but the discussion can be easily extended to other cases as well. Four streamlines with
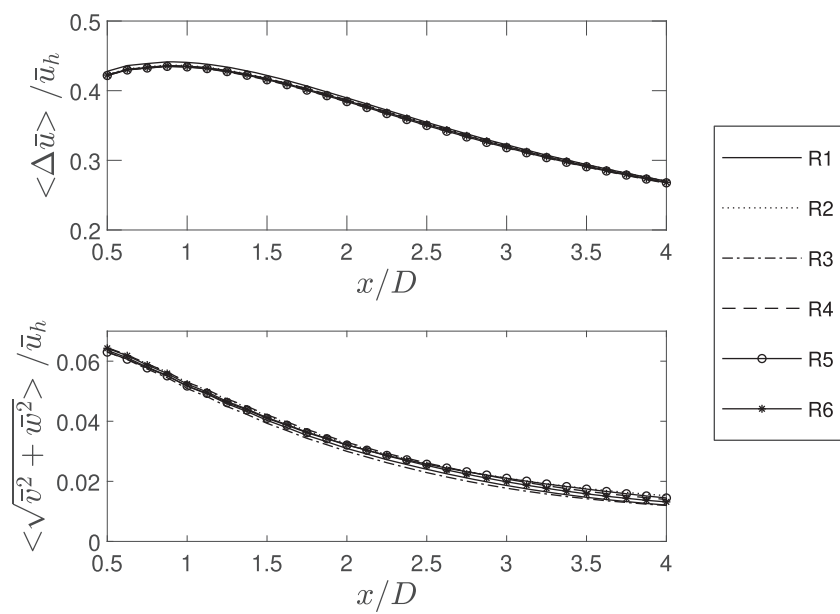

different starting points are depicted. Streamlines 1 and 2 are drawn to illustrate the strength of the wake rotation at different streamwise locations, while streamlines 3 and 4 help us to better understand flows above and beside the MR4 turbine, respectively.

As shown in Fig. 19(b), streamline 3 initially diverges from the wake (at $x<2 D$ ), but it then converges to the wake further downstream. The initial divergence of streamline 3 from the wake can be explained by the fact that at very short streamwise distances (e.g., $x=1 D$ ), as revealed in Fig. 19(a), the flow around the wake cross section moves outward due to flow blockage, despite movements induced by the wake rotation. In other words, blockage effects outweigh swirl motions and thus swirl motions can possibly enhance flow entrainment only further downstream (e.g., $x=3 D$ ) when blockage effects are negligible. However, at $x>2 D$, swirl motions induced by wake rotation are not anymore strong enough [see streamlines 1 and 2 in Fig. 19(a)] to substantially enhance the flow entrainment. For streamline 4, as seen in Fig. 19(c), both flow blockage and swirl motions due to wake rotation act in the same direction by moving the flow streamline away from the wake center.

It is worth mentioning that the presented results on the effect of wake rotation are related to one given rotor spacing (i.e., $s=0.1 d$ ). Therefore, whether the wake rotation has more important effects for

FIG. 18. Variations of the normalized velocity deficit $\Delta \bar{u} / \bar{u}_{h}$ (top panel) as well as cross-stream velocity magnitude $\sqrt{\bar{v}^{2}+\bar{w}^{2}} / \bar{u}_{h}$ (bottom panel), averaged over rotors, with streamwise distance for MR turbines with different rotation directions. Cases of $R 1-6$ are defined in Fig. 17. other values of rotor spacing remains an open question and can be addressed in future works. Moreover, it is important to note that in very large wind farms, vertical turbulent kinetic energy flux is the main mechanism of energy transport from the flow above into the 


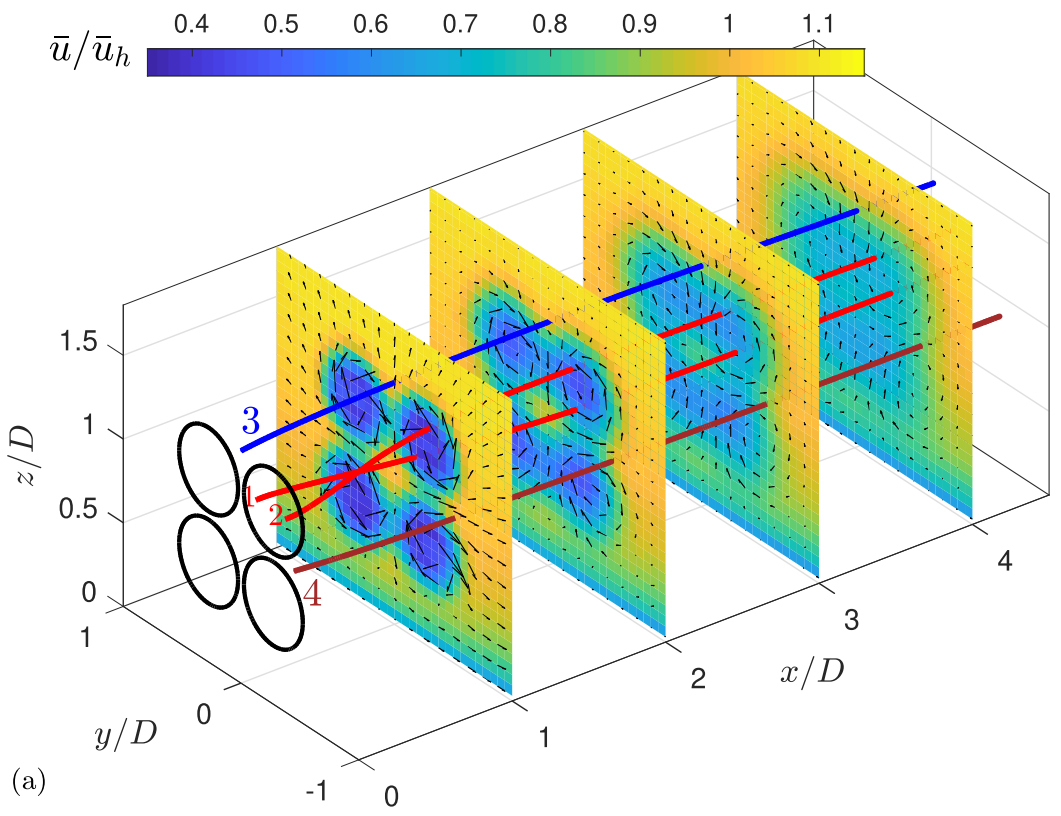

FIG. 19. (a) Contours of normalized velocity deficit overlaid by vector plots of cross-stream velocity components at different streamwise distances for case $R 6$ defined in Fig. 17. Color lines represent a few representative streamlines in the wake region. (b) Projection of streamline 3 on a vertical $x-z$ plane. (c) Projection of streamline 4 on a horizontal $x-y$ plane.
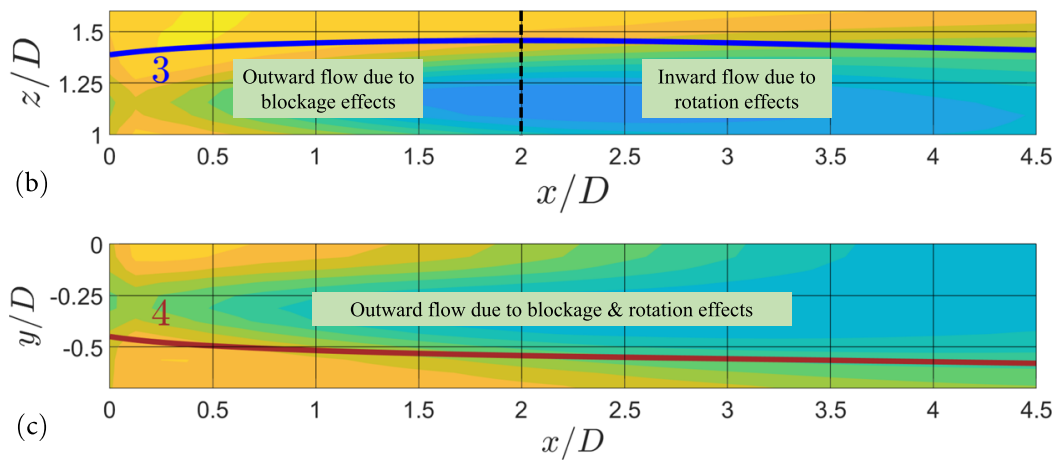

wind farm array. ${ }^{31,32,52,53}$ For large wind farms consisting of MR turbines, therefore, cases such as $R 4$ and $R 6$ might be of interest as they can generate an additional vertical flux of kinetic energy driven by mean swirl motions.

\section{SUMMARY AND CONCLUDING REMARKS}

A suite of LES in which turbine rotors are modeled as actuator disks was performed to study wake characteristics of MR turbines. Special focus is given to a detailed comparison of the wake of a SR turbine with the one of a MR turbine, whose geometrical arrangement is relatively similar to the Vestas multirotor turbine demonstrator. Our results show that, at first, the wake of the MR turbine consists of localized and high velocity-deficit regions downwind of each rotor. In comparison with the SR case, the maximum velocity deficit is found to be fairly smaller immediately behind each rotor of the MR turbine, which is arguably due to local flow blockage caused by adjacent rotors. It is also found that the MR turbine wake initially recovers faster than the one of the SR turbine although this fast recovery lasts only until wakes of multirotors mix and form a single wake. The mean kinetic energy budget analysis was employed to elucidate the effect of energy transport due to turbulence production on the MR turbine wake transition to a single wake. This analysis underpins the pivotal impact of this transition upon the recovery of MR turbine wakes.

The LES results of MR turbine wakes for different values of rotor spacing showed that wakes of MR turbines with larger rotor spacing are characterized by lower values of velocity deficit. This was explained by the fact that the increase in rotor spacing postpones the wake transition to a single wake. Using the analytical Gaussian wake model, a simple relationship was developed that can estimate the transition length for a MR turbine as a function of rotor spacing and inflow conditions. In addition to rotor spacing, the effect of rotor numbers on MR turbine wakes was examined in this paper. Our results show that increasing the number of rotors (and thereby reducing the size of rotors) has two opposing effects on the recovery of MR turbine wakes. Due to the smaller size of rotors, the wake of each individual rotor recovers faster given its shorter cross-stream 
length scale. However, a higher number of rotors makes the whole wake flow distribution more uniform which leads to an earlier transition of the wake array to a single wake. The effect of the rotation direction of rotors on wake flow distribution is also examined. Our results show that the distribution of the rotation direction of rotors cannot drastically alter the wake recovery for the studied MR turbine. Swirl motions caused by rotation of rotors cannot lead to flow entrainment through all the wake borders. Flow entrainment from a given direction (e.g., from above) can be achieved at the expense of losing momentum from another side. More importantly, movements due to the wake rotation are found to be overshadowed by the omnidirectional outward flow caused by flow blockage at short downwind distances.

Overall, consistent with the prior studies, ${ }^{12,13}$ our results highlight that the use of MR turbines can mitigate wake effects in wind farms. In comparison with the SR turbine wake, all of the tested MR turbine wakes in this paper were associated with lower values of velocity deficit and turbulence intensity at streamwise locations where a downstream turbine is usually installed (i.e., 5-8D). However, note that the advantage of MR turbine wakes over SR turbine ones is less plausible when the incoming flow is highly turbulent, considering the earlier transition of the wake array to a single wake in this case. More research on the effect of incoming turbulence on MR turbine wakes should be thus performed in future works. Moreover, further research is needed to study near wakes of multirotor turbines and their complex vortex dynamics. For instance, effects of adjacent rotors on the stability of tip vortices can be fruitfully explored by conducting either laboratory experiments or numerical simulations using high-resolution actuator-line techniques and advanced vortex methods.

\section{ACKNOWLEDGMENTS}

The authors acknowledge the useful comments by the anonymous reviewers.

\section{REFERENCES}

${ }^{1}$ P. Chasapogiannis, J. M. Prospathopoulos, S. G. Voutsinas, and T. K. Chaviaropoulos, "Analysis of the aerodynamic performance of the multi-rotor concept,” J. Phys.: Conf. Ser. 524(1), 012084 (2014).

${ }^{2}$ P. Jamieson, T. Chaviaropoulos, S. Voutsinas, M. Branney, G. Sieros, and P. Chasapogiannis, "The structural design and preliminary aerodynamic evaluation of a multi-rotor system as a solution for offshore systems of $20 \mathrm{MW}$ or more unit capacity," in EWEC and Exhibition Barcelona (EWEA, 2014), Vol. 1, PO.ID 203.

${ }^{3}$ P. Jamieson and M. Branney, "Multi-rotors; a solution to $20 \mathrm{MW}$ and beyond?," Energy Procedia 24, 52-59 (2012).

${ }^{4} \mathrm{P}$. Jamieson and M. Branney, "Structural considerations of a $20 \mathrm{MW}$ multi-rotor wind energy system," J. Phys.: Conf. Ser. 555(1), 012013 (2014).

${ }^{5} \mathrm{P}$. Verma, "Multi rotor wind turbine design and cost scaling," M.Sc. thesis, University of Massachusetts Amherst, 2014.

${ }^{6}$ S. N. S. Sandhu, "Comparative analysis of conventional and multi-rotor wind turbines,” Int. J. Circuits, Syst. Signal Process. 12, 246-255 (2018).

${ }^{7}$ N. S. Sandhu and S. Chanana, "Performance and economic analysis of multi-rotor wind turbine," EMITTER Int. J. Eng. Technol. 6(2), 289-316 (2018).

${ }^{8}$ Y. Zhou, R. So, M. Liu, and H. Zhang, "Complex turbulent wakes generated by two and three side-by-side cylinders," Int. J. Heat Fluid Flow 21(2), 125-133 (2000).
${ }^{9}$ M. M. Alam, Y. Zhou, and X. Wang, "The wake of two side-by-side square cylinders,” J. Fluid Mech. 669, 432-471 (2011).

${ }^{10}$ A. Rosenberg, S. Selvaraj, and A. Sharma, "A novel dual-rotor turbine for increased wind energy capture,” J. Phys.: Conf. Ser. 524(1), 012078 (2014).

${ }^{11}$ Z. Wang, W. Tian, A. Ozbay, A. Sharma, and H. Hu, "An experimental study on the aeromechanics and wake characteristics of a novel twin-rotor wind turbine in a turbulent boundary layer flow,” Exp. Fluids 57(9), 150 (2016).

${ }^{12}$ N. S. Ghaisas, A. S. Ghate, and S. K. Lele, "Large-eddy simulation study of multirotor wind turbines,” J. Phys.: Conf. Ser. 1037(7), 072021 (2018).

${ }^{13}$ M. P. van der Laan, S. J. Andersen, N. Ramos García, N. Angelou, G. R. Pirrung, S. Ott, M. Sjöholm, K. H. Sørensen, J. X. Vianna Neto, M. Kelly, T. K. Mikkelsen, and G. C. Larsen, "Power curve and wake analyses of the Vestas multi-rotor demonstrator," Wind Energy Sci, 4, 251-271 (2019).

${ }^{14}$ E. Bou-Zeid, C. Meneveau, and M. Parlange, "A scale-dependent Lagrangian dynamic model for large eddy simulation of complex turbulent flows," Phys. Fluids 17(2), 025105 (2005).

${ }^{15}$ R. Stoll and F. Porté-Agel, "Dynamic subgrid-scale models for momentum and scalar fluxes in large-eddy simulations of neutrally stratified atmospheric boundary layers over heterogeneous terrain," Water Resour. Res. 42(1), W01409, https://doi.org/10.1029/2005wr003989 (2006).

${ }^{16}$ F. Porté-Agel, Y. T. Wu, H. Lu, and R. J. Conzemius, "Large-eddy simulation of atmospheric boundary layer flow through wind turbines and wind farms," J. Wind Eng. Ind. Aerodyn. 99(4), 154-168 (2011).

${ }^{17} \mathrm{M}$. Abkar and F. Porté-Agel, "The effect of free-atmosphere stratification on boundary-layer flow and power output from very large wind farms," Energies 6 , 2338-2361 (2013).

${ }^{18} \mathrm{M}$. Abkar and F. Porté-Agel, "Influence of atmospheric stability on wind turbine wakes: A large-eddy simulation study,” Phys. Fluids 27(3), 035104 (2015).

${ }^{19}$ M. Abkar, J. Sørensen, and F. Porté-Agel, "An analytical model for the effect of vertical wind veer on wind turbine wakes," Energies 11(7), 1838 (2018).

${ }^{20}$ X. I. Yang and M. Abkar, "A hierarchical random additive model for passive scalars in wall-bounded flows at high Reynolds numbers," J. Fluid Mech. 842, 354380 (2018).

${ }^{21}$ M. Abkar, A. Sharifi, and F. Porté-Agel, "Wake flow in a wind farm during a diurnal cycle," J. Turbul. 17(4), 420-441 (2016).

${ }^{22} \mathrm{M}$. Abkar, "Impact of subgrid-scale modeling in actuator-line based largeeddy simulation of vertical-axis wind turbine wakes," Atmosphere 9(7), 257 (2018).

${ }^{23}$ A. E. Y1lmaz and J. Meyers, "Optimal dynamic induction control of a pair of inline wind turbines," Phys. Fluids 30(8), 085106 (2018).

${ }^{24} \mathrm{Y}$. T. Wu and F. Porté-Agel, "Large-eddy simulation of wind-turbine wakes: Evaluation of turbine parametrisations," Boundary-Layer Meteorol. 138(3), 345366 (2011).

${ }^{25}$ F. Porté-Agel, Y. T. Wu, and C. H. Chen, "A numerical study of the effects of wind direction on turbine wakes and power losses in a large wind farm," Energies 6(10), 5297-5313 (2013).

${ }^{26}$ Y. T. Wu and F. Porté-Agel, "Modeling turbine wakes and power losses within a wind farm using LES: An application to the Horns Rev offshore wind farm," Renewable Energy 75, 945-955 (2015).

${ }^{27}$ C. Garrett and P. Cummins, "The efficiency of a turbine in a tidal channel," J. Fluid Mech. 588, 243-251 (2007).

${ }^{28}$ G. T. Houlsby, S. Draper, and M. L. G. Oldfield, “Application of linear momentum actuator disc theory to open channel flow," Report No. OUEL 2296/08, 2008. ${ }^{29}$ T. Nishino and R. H. J. Willden, "The efficiency of an array of tidal turbines partially blocking a wide channel," J. Fluid Mech. 708, 596-606 (2012).

${ }^{30}$ T. Nishino and S. Draper, "Local blockage effect for wind turbines," J. Phys.: Conf. Ser. 625(1), 012010 (2015).

${ }^{31}$ M. Calaf, C. Meneveau, and J. Meyers, "Large eddy simulation study of fully developed wind turbine array boundary layers," Phys. Fluids 22, 015110 (2010).

${ }^{32}$ R. B. Cal, J. Lebrón, L. Castillo, H. S. Kang, and C. Meneveau, "Experimental study of the horizontally averaged flow structure in a model wind-turbine array boundary layer," J. Renewable Sustainable Energy 2(1), 013106 (2010).

${ }^{33}$ J. Meyers and C. Meneveau, "Flow visualization using momentum and energy transport tubes and applications to turbulent flow in wind farms," J. Fluid Mech. 715, 335-358 (2013). 
${ }^{34} \mathrm{M}$. Abkar and F. Porté-Agel, "Mean and turbulent kinetic energy budgets inside and above very large wind farms under conventionally-neutral condition," Renewable Energy 70, 142-152 (2014).

${ }^{35} \mathrm{D}$. Allaerts and J. Meyers, "Large eddy simulation of a large wind-turbine array in a conventionally neutral atmospheric boundary layer," Phys. Fluids 27(6), 065108 (2015).

${ }^{36} \mathrm{M}$. Abkar and F. Porté-Agel, "Influence of the Coriolis force on the structure and evolution of wind turbine wakes," Phys. Rev. Fluids 1, 063701 (2016).

${ }^{37}$ G. Cortina, M. Calaf, and R. B. Cal, "Distribution of mean kinetic energy around an isolated wind turbine and a characteristic wind turbine of a very large wind farm," Phys. Rev. Fluids 1(7), 074402 (2016).

${ }^{38}$ N. Ali, N. Hamilton, M. Calaf, and R. B. Cal, "Turbulence kinetic energy budget and conditional sampling of momentum, scalar, and intermittency fluxes in thermally stratified wind farms," J. Turbul. 20(1), 32-63 (2019).

${ }^{39}$ S. Pope, Turbulent Flows (Cambridge University Press, 2000).

${ }^{40} \mathrm{~A}$. Rosen and Y. Sheinman, "The power fluctuations of a wind turbine," J. Wind Eng. Ind. Aerodyn. 59, 51-68 (1996).

${ }^{41} \mathrm{~L}$. Chamorro and F. Porté-Agel, "A wind-tunnel investigation of wind-turbine wakes: Boundary-layer turbulence effects," Boundary-Layer Meteorol. 132, 129149 (2009).

${ }^{42} \mathrm{M}$. Bastankhah and F. Porté-Agel, "A new miniature wind turbine for wind tunnel experiments. Part I: Design and performance,” Energies 10(7), 908 (2017).

${ }^{43} \mathrm{M}$. Bastankhah and F. Porté-Agel, "A new miniature wind turbine for wind tunnel experiments. Part II: Wake structure and flow dynamics," Energies 10(7), 923 (2017).
${ }^{44}$ R. J. Stevens, D. F. Gayme, and C. Meneveau, "Effects of turbine spacing on the power output of extended wind-farms," Wind Energy 19(2), 359-370 (2016).

${ }^{45}$ N. Ali, N. Hamilton, D. DeLucia, and R. Bayoán Cal, "Assessing spacing impact on coherent features in a wind turbine array boundary layer," Wind Energy Sci. 3, 43-56 (2018)

${ }^{46} \mathrm{M}$. Bastankhah and F. Porté-Agel, "A new analytical model for wind-turbine wakes," Renewable Energy 70, 116-123 (2014).

${ }^{47}$ A. Niayifar and F. Porté-Agel, "Analytical modeling of wind farms: A new approach for power prediction,” Energies 9(9), 741 (2016).

${ }^{48}$ M. P. van der Laan and M. Abkar, "Improved energy production of multi-rotor wind farms," J. Phys.: Conf. Ser. 1256, 012011 (2019).

${ }^{49} \mathrm{M}$. Bastankhah and F. Porté-Agel, "Experimental and theoretical study of wind turbine wakes in yawed conditions," J. Fluid Mech. 806, 506-541 (2016).

${ }^{50}$ L. J. Vermeer, J. N. Sørensen, and A. Crespo, "Wind turbine wake aerodynamics," Prog. Aerosp. Sci. 39, 467-510 (2003).

${ }^{51} \mathrm{M}$. Bastankhah and F. Porté-Agel, "Wind tunnel study of the wind turbine interaction with a boundary-layer flow: Upwind region, turbine performance, and wake region," Phys. Fluids 29(6), 065105 (2017).

${ }^{52} \mathrm{C}$. VerHulst and C. Meneveau, "Altering kinetic energy entrainment in large eddy simulations of large wind farms using unconventional wind turbine actuator forcing," Energies 8(1), 370-386 (2015).

${ }^{53} \mathrm{C}$. Meneveau, "Big wind power: Seven questions for turbulence research," J. Turbul. 20(1), 2-20 (2019). 